B. Abdellaoui $\cdot$ E. Colorado $\cdot$ I. Peral

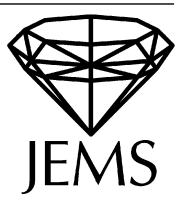

\title{
Existence and nonexistence results for a class of linear and semilinear parabolic equations related to some Caffarelli-Kohn-Nirenberg inequalities
}

\author{
Dedicated to Professor James Serrin on his seventy-sixth birthday
}

Received December 11, 2002 / final version received May 17, 2003

Abstract. In this work we study the problem

$$
\left\{\begin{array}{l}
u_{t}-\operatorname{div}\left(|x|^{-2 \gamma} \nabla u\right)=\lambda \frac{u^{\alpha}}{|x|^{2(\gamma+1)}}+f \text { in } \Omega \times(0, T), \\
u \geq 0 \text { in } \Omega \times(0, T), \quad u=0 \quad \text { on } \partial \Omega \times(0, T), \\
u(x, 0)=u_{0}(x) \text { in } \Omega,
\end{array}\right.
$$

$\Omega \subset \mathbb{R}^{N}(N \geq 2)$ is a bounded regular domain such that $0 \in \Omega, \lambda>0, \alpha>0,-\infty<\gamma<$ $(N-2) / 2, f$ and $u_{0}$ are positive functions such that $f \in L^{1}(\Omega \times(0, T))$ and $u_{0} \in L^{1}(\Omega)$. The main points under analysis are: (i) spectral instantaneous and complete blow-up related to the Harnack inequality in the case $\alpha=1,1+\gamma>0$; (ii) the nonexistence of solutions if $\alpha>1$, $1+\gamma>0$; (iii) a uniqueness result for weak solutions (in the distribution sense); (iv) further results on existence of weak solutions in the case $0<\alpha \leq 1$.

\section{Introduction}

We take as starting point the results by Baras and Goldstein $[\overline{\mathrm{BG}}]$. In $[\overline{\mathrm{PV}}]$ and $[\overline{\mathrm{BC}}]$ those results are related to a Hardy inequality. With this perspective we can see the results by Baras-Goldstein as a kind of spectral instantaneous and complete blow-up: roughly speaking, if we consider the heat equation with a singular perturbation, $u_{t}-\Delta u=$ $\lambda|x|^{-2} u$, then the initial-boundary value problem has a global solution for some large class of initial data if $\lambda\left\langle\lambda_{N, 0}:=(N-2)^{2} / 4\right.$, and has no positive solution for $\lambda \geq \lambda_{N, 0}$. In fact $\lambda_{N, 0}$ is the optimal constant in the Hardy inequality $(19)$ below, in the case $\gamma=0$. It is well known that $\lambda_{N, 0}$ is not attained. In $[\overline{\mathrm{AgP}}],[\mathrm{DGP}]$ and $[\mathrm{GP}]$, for some class of parabolic equations related to generalized Hardy-Sobolev inequalities, it was shown that the spectral instantaneous blow-up is not true, in the sense that even if the parameter $\lambda$

This work has been supported by Project BFM2001-0183 of M.C.Y.T. of Spain.

B. Abdellaoui, E. Colorado, I. Peral: Departamento de Matemáticas, Universidad Autónoma de Madrid, 28049 Madrid, Spain; e-mail: boumediene.abdellaoui@uam.es, eduardo.colorado@uam.es,ireneo.peral@uam.es

Mathematics Subject Classification (2000): 35K10, 35K15, 35K65, 35J70, 46E35 
is larger than the optimal constant in the corresponding Hardy-Sobolev inequality, there exists a solution. In this paper we study the following problem:

$$
\left\{\begin{array}{l}
u_{t}-\operatorname{div}\left(|x|^{-2 \gamma} \nabla u\right)=\lambda \frac{u^{\alpha}}{|x|^{2(\gamma+1)}}+f \quad \text { in } \Omega \times(0, T), \\
u \geq 0 \quad \text { in } \Omega \times(0, T), \quad u=0 \quad \text { on } \partial \Omega \times(0, T) \\
u(x, 0)=u_{0}(x) \text { in } \Omega
\end{array}\right.
$$

where $\Omega \subset \mathbb{R}^{N}(N \geq 2)$ is a bounded regular domain such that $0 \in \Omega, \lambda>0, \alpha>0$, $-\infty<\gamma<(N-2) / \overline{2}, f$ and $u_{0}$ are positive functions such that $f \in L^{1}(\Omega \times(0, T))$ and $u_{0} \in L^{1}(\Omega)$. Notice that the case $\gamma=0$ and $\alpha=1$ is just the problem solved by Baras and Goldstein in $[\mathrm{BG}]$. In the opposite direction, in $[\mathrm{DGP}]$ it is proved, in particular, that if $1+\gamma \leq 0$, problem (2) with $\alpha=1$ has a global weak solution for all $\lambda \in \mathbb{R}$, that is, the spectral instantaneous and complete blow-up does not occur. However, the corresponding Hardy-Sobolev inequality holds.

We will mainly discuss the case $1+\gamma>0$ related to the spectral instantaneous and complete blow-up. The fundamental difference with the case $1+\gamma \leq 0$ is that if $1+\gamma>0$, the associated homogeneous linear differential equation satisfies the parabolic Harnack inequality [10, proved by Chiarenza and Serapioni in [ChS] and by Gutiérrez and Wheeden in $[\mathrm{GW}]$ for more general cases of degenerate equations. Moreover the interval of $\gamma$ is optimal. More precisely, if $1+\gamma \leq 0$ we consider $u(x, t)=t|x|^{\rho}$ where $\rho>0$. A direct computation shows that

$$
u_{t}-\operatorname{div}\left(|x|^{-2 \gamma} \nabla u\right)=|x|^{\rho}-\rho t|x|^{\rho-2(\gamma+1)}(\rho+N-2(\gamma+1)) .
$$

As $\rho>0$ and $\gamma+1 \leq 0$, for $r_{0}, t_{0}>0$ small enough, $u$ is a supersolution to problem (9) in the cylinder $B_{r_{0}}(0) \times\left(0, t_{0}\right)$. Since $u(0, t)=0$, even the weak Harnack inequality, given by (12), is not satisfied. Then we discover that the Harnack inequality together with the Hardy-Sobolev inequality (19) yield the spectral instantaneous and complete blow-up. The case of $\gamma=0$ and $\alpha>1$, that is, the heat equation with a perturbation $u^{\alpha}|x|^{-2}$, was studied in $[\overline{\mathrm{BC}}]$, where it was shown that the complete and instantaneous blow-up is independent of $\lambda>0$, namely, problem (2) has no positive solutions even in the distribution sense apart from the trivial one.

The Sobolev space $\mathcal{D}_{0, \gamma}^{1,2}(\Omega)$ is defined as the completion of $C_{0}^{\infty}(\Omega)$ under the norm

$$
\|\phi\|_{\gamma}^{2}=\int_{\Omega}|x|^{-2 \gamma}|\nabla \phi|^{2} d x .
$$

It is clear that $\mathcal{D}_{0, \gamma}^{1,2}(\Omega)$ is a Hilbert space. Notice that $\mathcal{D}_{0, \gamma}^{1,2}(\Omega) \subset W_{0}^{1,2}(\Omega)$ for $\gamma>0$ and $W_{0}^{1,2}(\Omega) \subset \mathcal{D}_{0, \gamma}^{1,2}(\Omega)$ if $\gamma \leq 0$. This is the natural space to work in with the elliptic part and to look for solutions in the classical energy sense or, more precisely, solutions in a variational framework.

Next we specify in what more general sense we will consider solutions when the data of the problem are not variational.

First, following $[\overline{\mathrm{BM}}]$ and $[\overline{\mathrm{Pr}}]$, we define an entropy solution. As usual we will consider the truncation $T_{k}(u)=u-\operatorname{sign}(u)(|u|-k)_{+}$. 
Definition 1.1. We say that

$$
u \in \mathcal{T}_{0, \gamma}^{1,2}(\Omega \times(0, T)) \quad \text { if } \quad T_{k}(u) \in L^{2}\left((0, T) ; \mathcal{D}_{0, \gamma}^{1,2}(\Omega)\right) \text { for all } k>0
$$

Definition 1.2. Assume that $f$ and $u_{0}$ are positive functions such that $f \in L^{1}(\Omega \times$ $(0, T))$ and $u_{0} \in L^{1}(\Omega)$. A function $u \in C\left([0, T] ; L^{1}(\Omega)\right)$ is an entropy solution to the problem

$$
\left\{\begin{array}{l}
u_{t}-\operatorname{div}\left(|x|^{-2 \gamma} \nabla u\right)=f \quad \text { in } \Omega \times(0, T) \\
u=0 \text { on } \partial \Omega \times(0, T) \\
u(x, 0)=u_{0}(x) \quad \text { in } \Omega
\end{array}\right.
$$

if $u \in \mathcal{T}_{0, \gamma}^{1,2}(\Omega \times(0, T))$ and

$$
\begin{aligned}
\int_{\Omega} \Theta_{k}(u-v)(T) d x+ & \int_{0}^{T} \int_{\Omega} v_{t} T_{k}(u-v) d x d t \\
& +\int_{0}^{T} \int_{\Omega} \nabla u \nabla\left(T_{k}(u-v)\right)|x|^{-2 \gamma} d x d t \\
& =\int_{\Omega} \Theta_{k}\left(u_{0}-v(0)\right) d x+\int_{0}^{T} \int_{\Omega} f T_{k}(u-v) d x d t
\end{aligned}
$$

for all $v \in L^{2}\left((0, T) ; \mathcal{D}_{0, \gamma}^{1,2}(\Omega)\right) \cap L^{\infty}(\Omega \times[0, T]) \cap C\left([0, T] ; L^{1}(\Omega)\right)$ and for all $k>0$, where

$$
\Theta_{k}(s)=\int_{0}^{s} T_{k}(t) d t
$$

Notice that as $u \in C\left([0, T] ; L^{1}(\Omega)\right)$,

$$
\int_{\Omega} u_{t} T_{k}(u) d x=\frac{d}{d t}\left(\int_{\Omega} \Theta_{k}(u) d x\right),
$$

or

$$
\int_{\Omega} \Theta_{k}(u(t)) d x-\int_{\Omega} \Theta_{k}(u(0)) d x=\int_{0}^{t} \int_{\Omega} u_{s} T_{k}(u) d x d s .
$$

Using the same arguments as in $[\overline{\mathrm{BM}}]$ and $[\overline{\mathrm{Pr}}]$ it is easy to prove the following result.

Theorem 1.3. Let $\Omega$ be a bounded regular domain, and assume that $u_{0} \in L^{1}(\Omega)$ and $f \in L^{1}(\Omega \times(0, T))$ are positive functions. Then problem 3 has a unique positive entropy solution.

As a consequence we have the following comparison principle that we will use systematically.

Lemma 1.4. Let $u, v$ be entropy solutions of (3) with data $f, u_{0}$ and $g$, $v_{0}$ respectively. Suppose that $0 \leq g \leq f$ and $0 \leq v_{0} \leq u_{0}$. Then $0 \leq v \leq u$.

On the other hand, we have the following definition of weak solution. 
Definition 1.5. Let $f(x, t)$ be a function in $L^{1}(\Omega \times(0, T))$ and let $u_{0} \in L^{1}(\Omega)$. A function $u \in C\left([0, T) ; L^{1}\left(\left(1+|x|^{-2 \gamma-1}\right) d x\right)\right)$ is a weak solution to

$$
\left\{\begin{array}{l}
u_{t}-\operatorname{div}\left(|x|^{-2 \gamma} \nabla u\right)=f(x, t), \\
u=0 \text { on } \partial \Omega \times(0, T), \\
u(x, 0)=u_{0}(x) \quad \text { in } L^{1}(\Omega),
\end{array}\right.
$$

if for all $0<s<T$ we have

$$
\int_{0}^{s} \int_{\Omega} u\left(-\psi_{t}-\operatorname{div}\left(|x|^{-2 \gamma} \nabla \psi\right)\right)-\int_{\Omega} u_{0} \psi(0)=\int_{0}^{s} \int_{\Omega} f \psi d x d t
$$

for all $\xi \in C^{2}(\Omega \times[0, s])$ with $\xi(x, s)=0$ on $\Omega$ and $\xi=0$ on $\partial \Omega \times[0, s]$.

We write $u(x, 0)=u_{0}$ meaning that $\lim _{t \rightarrow 0} \int_{\Omega}\left|u(x, t)-u_{0}(x)\right| d x=0$.

In Section 3 we will prove the following result on uniqueness.

Theorem 1.6. Let $-\infty<\gamma<(N-2) / 2$. Assume that $u$ is a weak solution to the problem

$$
\left\{\begin{array}{l}
u_{t}-\operatorname{div}\left(|x|^{-2 \gamma} \nabla u\right)=0, \\
u=0 \text { on } \partial \Omega \times(0, T), \\
u(x, 0)=0 \text { in } L^{1}(\Omega)
\end{array}\right.
$$

Then $u \equiv 0$.

To prove the uniqueness Theorem 1.6 we require a Weyl type result for the associated elliptic equation proved in [AP3]. For the reader's convenience, we sketch the proof in the Appendix. In consequence of this uniqueness result all the arguments will be done by approximation, understood in a convenient way.

The paper is organized as follows. In Section 2 we state the Harnack inequality (see $[\mathrm{ChS}]$ ) and we give the weak version which we use in the paper. We also state the HardySobolev inequality for weights, as a particular case of the Cafarelli-Kohn-Nirenberg inequalities. Finally, we formulate a consequence of a Picone identity (see $[\overline{\mathrm{P}}]$ ), that will be an important tool in the proofs of blow-up. Section 3 deals with the proof of Theorem 1.6. Section 4 is devoted to proving the main results about blow-up for $1+\gamma>0$. In the first subsection we study the spectral complete and instantaneous blow-up in the linear case (Theorems 4.1 and 4.6. The second subsection deals with the superlinear case; here without any restriction on $\lambda$, we are able to prove the nonexistence result (Theorem 4.77), and the complete blow-up for any positive data (Theorem 4.10). In Section 5 we study the sublinear case: we prove the existence of a global solution if the initial data belongs to $L^{2}(\Omega)$ (Theorem 5.1) and for more general initial data (Theorem 5.5). Moreover, we obtain the asymptotic behavior of the solutions as $t \rightarrow \infty$, if the initial data is less than or equal to the stationary solution. In Section 6 we state the existence results proved in [DGP] for the case $1+\gamma \leq 0$ to compare them with the case $1+\gamma>0$. Finally, in the Appendix we prove a result of regularity (and then uniqueness) of Weyl type for the associated elliptic equation. 


\section{Preliminaries}

The Harnack inequality for parabolic equations will be an important tool in our discussion. Let $R=B_{\rho}\left(x_{0}\right) \times\left(t_{0}-\beta, t_{0}+\beta\right) \subset \Omega \times(0, T), 0<\beta<t_{0}$. Consider the equation

$$
u_{t}-\operatorname{div}\left(|x|^{-2 \gamma} \nabla u\right)=0 \quad \text { in } R,
$$

where $1+\gamma>0$. We say that $u \in L^{2}\left((0, T) ; \mathcal{D}_{0, \gamma}^{1,2}(\Omega)\right) \cap C\left([0, T] ; L^{1}(\Omega)\right)$ is an energy solution to 99 if

$$
\int_{0}^{T} \int_{\Omega} v_{t} u+\int_{0}^{T} \int_{\Omega}|x|^{-2 \gamma} \nabla u \nabla v=0
$$

for all $v \in L^{2}\left((0, T) ; \mathcal{D}_{0, \gamma}^{1,2}(\Omega)\right) \cap C\left([0, T] ; L^{1}(\Omega)\right)$. Below, sup and inf will denote the essential supremum and essential infimum respectively. The concrete result that we use is the following.

Theorem 2.1 (Harnack Inequality). Let $u$ be a positive energy solution to (9) with $1+\gamma>0$. Then there exists $C=C\left(N, \gamma, \rho, t_{0}, \beta\right)$ such that

$$
\sup _{R^{-}} u \leq C \inf _{R^{+}} u,
$$

where $R^{-}=B_{\rho / 2}\left(x_{0}\right) \times\left(t_{0}-\frac{3}{4} \beta, t_{0}-\frac{1}{4} \beta\right), R^{+}=B_{\rho / 2}\left(x_{0}\right) \times\left(t_{0}+\frac{1}{4} \beta, t_{0}+\beta\right)$.

For a proof, we refer to [ChS] and also [GW] for some extensions. We will use the following weak Harnack inequality for positive supersolutions.

Theorem 2.2. Let $u \in L^{2}\left((0, T) ; \mathcal{D}_{0, \gamma}^{1,2}(\Omega)\right) \cap C\left([0, T] ; L^{1}(\Omega)\right)$ be a positive supersolution to problem (9) with $1+\gamma>0$. Then there exists a positive constant $C=$ $C\left(N, \gamma, \rho, t_{0}, \beta\right)$ such that

$$
\int_{B_{\rho / 2}\left(x_{0}\right)} u(x, t) d x \leq C \inf _{R^{+}} u \quad \text { for all } t \in\left(t_{0}-\frac{3}{4} \beta, t_{0}-\frac{1}{4} \beta\right) .
$$

Moreover

$$
\iint_{R^{-}} u(x, t) d x d t \leq C \inf _{R^{+}} u
$$

Proof. Since $u \in C\left([0, T] ; L^{1}(\Omega)\right)$, there exists $\bar{t} \in\left[t_{0}-\frac{3}{4} \beta, t_{0}-\frac{1}{4} \beta\right]$ such that

$$
\sup _{t \in\left(t_{0}-\frac{3}{4} \beta, t_{0}-\frac{1}{4} \beta\right)} \int_{B_{\rho / 2}\left(x_{0}\right)} u(x, t) d x=\int_{B_{\rho / 2}\left(x_{0}\right)} u(x, \bar{t}) d x .
$$

Let $v$ be the solution to the problem

$$
\left\{\begin{array}{l}
v_{t}-\operatorname{div}\left(|x|^{-2 \gamma} \nabla v\right)=0 \quad \text { in } \Omega \times\left(\bar{t}, t_{0}+\beta\right), \\
v(x, \bar{t})=u(x, \bar{t}), \quad x \in \Omega
\end{array}\right.
$$

By comparison, $u \geq v$. Let $\xi$ be the positive solution to the adjoint problem

$$
\left\{\begin{array}{l}
-\xi_{t}-\operatorname{div}\left(|x|^{-2 \gamma} \nabla \xi\right)=h \quad \text { in } \Omega \times\left(\bar{t}, t_{0}+\beta\right), \\
\xi\left(x, t_{0}+\beta\right)=0, \quad x \in \Omega
\end{array}\right.
$$


where $h$ is a bounded positive function that will be chosen later. By the maximum principle, $\xi \geq 0$. Using $\xi$ as a test function in (14), we obtain

$$
-\int_{\Omega} \xi(x, \bar{t}) v(x, \bar{t}) d x+\int_{\bar{t}}^{t_{0}+\beta} \int_{\Omega} v\left(-\xi_{t}-\operatorname{div}\left(|x|^{-2 \gamma} \nabla \xi\right)\right) d x d t=0
$$

therefore

$$
\begin{aligned}
\int_{\bar{t}}^{t_{0}+\beta} \int_{\Omega} v(x, t) h(x, t) d x d t & =\int_{\Omega} u(x, \bar{t}) \xi(x, \bar{t}) d x \\
& \geq \min _{B_{\rho / 2}\left(x_{0}\right)} \xi(x, \bar{t}) \int_{B_{\rho / 2}\left(x_{0}\right)} u(x, \bar{t}) d x
\end{aligned}
$$

By choosing $h=\chi_{B_{\rho / 2}\left(x_{0}\right)} \chi_{\left(t_{0}+\frac{1}{4} \beta, t_{0}+\beta\right)}$, we conclude that

$$
\iint_{R^{+}} v(x, t) d x d t \geq \min _{B_{\rho / 2}\left(x_{0}\right)} \xi(x, \bar{t}) \int_{B_{\rho / 2}\left(x_{0}\right)} u(x, \bar{t}) d x .
$$

Finally, by Theorem 2.1 applied to $v$ and Lemma 1.4 , we obtain

$$
\sup _{R^{-}} v \leq c \inf _{R^{+}} v \leq c \inf _{R^{+}} u
$$

From (13), 16) and (17), we conclude that

$$
\iint_{R^{-}} u(x, t) \leq c_{1} \int_{B_{\rho / 2}\left(x_{0}\right)} u(x, \bar{t}) d x \leq c_{2} \inf _{R^{+}} u,
$$

where $c_{1}, c_{2}$ are independent on $u$.

This last result is an important fact to analyze the blow-up behavior. Notice that the homogeneous parabolic equation associated to problem (2) satisfies the weak Harnack inequality $(12)$ if and only if $1+\gamma>0$, according to the counterexample explained in the introduction, i.e., the function $u(x, t)=t|x|^{\rho}$ where $\rho>0$.

If $f \geq 0$, then we can prove the following extension of the Harnack inequality to entropy solutions.

Corollary 2.3. Let $f \in L^{1}(\Omega \times(0, T))$ be such that $f \geq 0$. Assume that $u$ is a positive entropy solution to problem (3) with $1+\gamma>0$. Then there exists a positive constant $C=C\left(N, \gamma, \rho, t_{0}, \beta\right)$ such that

$$
\sup _{t \in\left(t_{0}-\frac{3}{4} \beta, t_{0}-\frac{1}{4} \beta\right)} \int_{B_{\rho / 2}\left(x_{0}\right)} u(x, t) d x \leq C \inf _{R^{+}} u
$$

Notice that as a consequence we get the strong maximum principle for positive entropy solutions if $f \geq 0$. 
Remark 2.4. In the interval $0<1+\gamma<N / 2$, for which we have the parabolic Harnack inequality, we are able to calculate with an elementary argument of homogeneity the gaussian-like selfsimilar solution,

$$
E(x, t)=C \frac{H(t)}{t^{N / 2(\gamma+1)}} e\left(-\frac{|x|^{2(\gamma+1)}}{4(\gamma+1)^{2} t}\right),
$$

where $H$ is the Heaviside function. Normalizing the constant $C$ we realize that

$$
E_{t}-\operatorname{div}\left(|x|^{-2 \gamma} \nabla E\right)=\delta_{(0,0)} .
$$

For $u \in \mathcal{D}_{0, \gamma}^{1,2}(\Omega)$ we have the following Caffarelli-Kohn-Nirenberg inequalities (see $[\overline{C K N}]$ ).

Proposition 2.5 (Caffarelli-Kohn-Nirenberg). Let $p, q, r, \alpha, \beta, \sigma$ and a be real constants such that $p, q \geq 1, r>0,0 \leq a \leq 1$, and

$$
\frac{1}{p}+\frac{\alpha}{N}, \frac{1}{q}+\frac{\beta}{N}, \frac{1}{r}+\frac{m}{N}>0,
$$

where $m=a \sigma+(1-a) \beta$. Then there exists a positive constant $C$ such that

$$
\left\||x|^{m} u\right\|_{L^{r}\left(\mathbb{R}^{N}\right)} \leq C\left\||x|^{\alpha}|\nabla u|\right\|_{L^{p}\left(\mathbb{R}^{N}\right)}^{a}\left\||x|^{\beta} u\right\|_{L^{q}\left(\mathbb{R}^{N}\right)}^{1-a}
$$

for all $u \in C_{0}^{\infty}\left(\mathbb{R}^{N}\right)$ if and only if the following relations hold:

$$
\begin{aligned}
\frac{1}{r}+\frac{m}{N} & =a\left(\frac{1}{p}+\frac{\alpha-1}{N}\right)+(1-a)\left(\frac{1}{q}+\frac{\beta}{N}\right) . \\
\alpha-\sigma & \geq 0 \quad \text { if } a>0, \\
\alpha-\sigma & \leq 1 \quad \text { if } a>0 \text { and } \frac{1}{r}+\frac{m}{N}=\frac{1}{p}+\frac{\alpha-1}{N} .
\end{aligned}
$$

In particular we get the following extension of the Hardy-Sobolev inequality (see also $[\mathrm{CW}])$

Lemma 2.6. Let $N \geq 2$ and $-\infty<\gamma<(N-2) / 2$. Then for all $u \in \mathcal{D}_{0, \gamma}^{1,2}(\Omega)$,

$$
\lambda_{N, \gamma} \int_{\mathbb{R}^{N}} \frac{|u|^{2}}{|x|^{2(\gamma+1)}} d x \leq \int_{\mathbb{R}^{N}} \frac{|\nabla u|^{2}}{|x|^{2 \gamma}} d x, \quad \lambda_{N, \gamma}=\left(\frac{N-2(\gamma+1)}{2}\right)^{2} .
$$

Moreover $\lambda_{N, \gamma}$ is optimal and it is not attained.

The following Sobolev inequality will be used in the last section to prove the behavior of solutions.

Theorem 2.7 (Sobolev Inequality). There exists a positive constant $C=C(N, \gamma)$ such that for all $u \in \mathcal{D}_{0, \gamma}^{1,2}(\Omega)$,

$$
\left(\int_{\Omega}|u|^{2^{*}}|x|^{-2^{*} \gamma} d x\right)^{1 / 2^{*}} \leq C\left(\int_{\Omega}|\nabla u|^{2}|x|^{-2 \gamma} d x\right)^{1 / 2},
$$

where $2^{*}=\frac{2 N}{N-2}$. 
Finally, an important tool to prove the nonexistence is the following theorem whose proof can be found in [AP3].

Theorem 2.8 (Picone Inequality). Let $u, v \in \mathcal{D}_{0, \gamma}^{1,2}(\Omega)$ with $v>0$ in $\Omega$, and suppose that $-\operatorname{div}\left(|x|^{-2 \gamma} \nabla v\right)$ is a positive bounded Radon measure. Then

$$
\int_{\Omega}|x|^{-2 \gamma}|\nabla u|^{2} d x \geq \int_{\Omega} \frac{-\operatorname{div}\left(|x|^{-2 \gamma} \nabla v\right)}{v} u^{2} d x .
$$

\section{A result on uniqueness}

The main result of this part is the following uniqueness theorem which is true for all $\gamma \in(-\infty,(N-2) / 2)$. The proof is deeply inspired by the papers by Benilan-BrezisCrandall $[\overline{\mathrm{BBC}}]$, Brezis-Crandall $[\overline{\mathrm{BCr}}]$ and Pierre $[\overline{\mathrm{Pi}}]$. An important tool in the proof is a regularity result for the associated elliptic equation obtained in [AP3], with the same flavor as the classical result by Weyl for the Laplace operator. (See the Appendix at the end of this work.)

Theorem 3.1. Assume that $u$ is a weak solution (in the sense of Definition 1.5) to the problem

$$
\left\{\begin{array}{l}
u_{t}-\operatorname{div}\left(|x|^{-2 \gamma} \nabla u\right)=0 \\
u=0 \text { on } \partial \Omega \times(0, T) \\
u(x, 0)=0 \quad \text { in } L^{1}(\Omega)
\end{array}\right.
$$

Then $u \equiv 0$.

Proof. To simplify the notation we set $L_{\gamma} v=\operatorname{div}\left(|x|^{-2 \gamma} \nabla v\right)$. Let $u$ be a weak solution to 21. We claim that

$$
u(t)-u(s)=L_{\gamma} \int_{s}^{t} u(\sigma) d \sigma \quad \text { in } \mathcal{D}^{\prime}(\Omega) .
$$

To prove the claim we set $X(t)=\int_{\Omega} u(x, t) \phi(x) d x$, where $t \in(0, T)$ and $\phi \in C_{0}^{\infty}(\Omega)$. Notice that $X \in L^{1}(0, T)$. Let $\alpha \in C_{0}^{\infty}(0, T)$. Then

$$
\begin{aligned}
\left\langle X^{\prime}, \alpha\right\rangle & \equiv-\left\langle X, \alpha^{\prime}\right\rangle \\
& =-\int_{0}^{T} \int_{\Omega} u(x, t) \phi(x) \alpha^{\prime}(t) d x d t=\int_{0}^{T} \int_{\Omega} u(x, t) \alpha(t) L_{\gamma}(\phi(x)) d x d t .
\end{aligned}
$$

Hence

$$
X^{\prime}(t)=\int_{\Omega} u(x, t) L_{\gamma}(\phi(x)) d x \quad \text { in } \mathcal{D}^{\prime}(0, T) .
$$

Since $\int_{\Omega} u(x, t) L_{\gamma}(\phi(x)) d x \in L^{1}(0, T)$, integrating 22 we obtain the claim.

As $\int_{\Omega}|u(x, s)| d x \rightarrow 0$ as $s \rightarrow 0$ we deduce that

$$
u(t)=L_{\gamma} \int_{0}^{t} u(\sigma) d \sigma \quad \text { in } \mathcal{D}^{\prime}(\Omega) .
$$


Let $v(t)$ be the weak solution to the elliptic problem

$$
-L_{\gamma} v(t)=u(t),\left.\quad v(t)\right|_{\partial \Omega}=0 .
$$

By the uniqueness result (Corollary 7.4 in the Appendix), since $u(t) \in L^{1}(\Omega)$, it follows that $v$ coincides with the entropy solution to the elliptic problem [24]. (See [ब्AP3] for the definition and properties of entropy solutions in this concrete case of elliptic equations.) Hence we conclude that $v(t)=-\int_{0}^{t} u(\sigma) d \sigma$. Thus $v$ is a differentiable function in $(0, T)$ and

$$
\frac{\partial v}{\partial t}(t)=u(t) \in L_{d \mu}^{1}(\Omega \times(0, T)), \quad d \mu=\left(|x|^{-2 \gamma-1}+1\right) d x d t .
$$

Therefore $v$ is a solution to the problem

$$
-L_{\gamma} v(t)=u(t)=-\frac{\partial v}{\partial t},\left.\quad v(t)\right|_{\partial \Omega}=0 .
$$

We can use $T_{k}(v)$ as a test function in 25 . Hence we get

$$
\int_{\Omega}\left|\nabla T_{k}(v)\right|^{2}|x|^{-2 \gamma} d x=-\int_{\Omega} \frac{\partial v}{\partial t} T_{k}(v) d x=-\frac{d}{d t}\left(\int_{\Omega} \Theta_{k}(v) d x\right),
$$

where $\Theta_{k}$ is defined in (5). By integration we obtain

$$
\int_{0}^{T} \int_{\Omega}\left|\nabla T_{k}(v)\right|^{2}|x|^{-2 \gamma} d x+\int_{0}^{T} \int_{\Omega} \Theta_{k}(v(T)) d x=0 .
$$

Since $\Theta_{k}(s) \geq 0$ we conclude that $\left|\nabla T_{k}(v)\right| \equiv 0$ for all $k>0$ and then $v \equiv 0$, which implies $u \equiv 0$.

\section{Remark 3.2.}

1) Notice that Theorem 3.1 and the linearity of the problem provide the uniqueness for problem (6).

2) By using the Caffarelli-Kohn-Nirenberg inequalities a direct calculation proves that the entropy solutions are weak solutions in the sense of Definition 1.5 . See the details in [AP3].

\section{Nonexistence results: Blow-up in the case $1+\gamma>0$}

Since we have the uniqueness Theorem 3.1, it is sufficient to work with entropy solutions, which means that we will have in mind the techniques and results for this kind of solutions. 
4.1. The linear case, $\alpha=1,1+\gamma>0$ and $\lambda>\lambda_{N, \gamma}$, spectral instantaneous and complete blow-up

This subsection deals with the following problem:

$$
\left\{\begin{array}{l}
u_{t}-\operatorname{div}\left(|x|^{-2 \gamma} \nabla u\right)=\lambda \frac{u}{|x|^{2(\gamma+1)}, \quad u \geq 0 \quad \text { in } \Omega \times(0, T),} \\
u=0 \quad \text { on } \partial \Omega \times(0, T), \quad u(x, 0)=u_{0}(x) \quad \text { in } \Omega,
\end{array}\right.
$$

where $u_{0}$ is a positive function such that $u_{0} \in L^{\infty}(\Omega)$, and $\lambda>\lambda_{N, \gamma}$. The main result about problem (26) is the following theorem.

Theorem 4.1. Let $u_{0} \in L^{\infty}(\Omega)$ be a positive function and assume that $\lambda>\lambda_{N, \gamma}$. If $u_{0} \neq 0$, then problem $(26)$ with $1+\gamma>0$ has no positive weak solution.

Proof. Let $u \geq 0$ be a positive weak solution to 26. By Corollary 2.3 we obtain $u>0$. We set

$$
\begin{aligned}
a_{n}(x) & =\frac{1}{|x|^{2(\gamma+1)}+1 / n}, \\
g_{n}(s) & =\min \{n, s\} .
\end{aligned}
$$

Let $u_{n}$ be the unique solution to the problem

$$
\left\{\begin{array}{l}
\left(u_{n}\right)_{t}-\operatorname{div}\left(|x|^{-2 \gamma} \nabla u_{n}\right)=\lambda a_{n}(x) g_{n}(u) \quad \text { in } \Omega \times(0, T), \\
u_{n}=0 \quad \text { on } \partial \Omega \times(0, T), \quad u_{n}(x, 0)=u_{0}(x) \quad \text { in } \Omega .
\end{array}\right.
$$

Notice that by the estimates in [ChF], $u_{n} \in L^{\infty}(\Omega \times(0, T))$. (See also [AP4] for details and more general results.) Since $u_{0} \neq 0$, using the Harnack inequality we find that $u_{n}>0$ in $\Omega \times(0, T)$ and $u_{n}$ converges to $u$ in $C\left([0, T] ; L^{1}(\Omega)\right)$. Since $\left\{a_{n}(x) g_{n}(u)\right\}$ is increasing with respect to $n$, it follows that $\left\{u_{n}\right\}$ is also increasing.

Using the Harnack inequality again we get

$$
u_{1} \geq \varepsilon \quad \text { a.e. in } B_{\eta}(0) \times(\tau, T)
$$

for some $0<\tau<T$. Since $u \geq u_{1}$, we have $u \geq \varepsilon$ a.e. in $B_{\eta}(0) \times(\tau, T)$. For $\bar{\varepsilon}>0$ we can choose a constant $c=c(\eta, p, \bar{\varepsilon})$ such that $v(x)=c(\log (1 /|x|)-\log (1 /|\eta|))$ satisfies

$$
\left\{\begin{array}{l}
v_{t}-\operatorname{div}\left(|x|^{-2 \gamma} \nabla v\right)=\frac{\bar{\varepsilon}}{|x|^{2(\gamma+1)}} \quad \text { in } B_{\eta}(0) \times(\tau, T), \\
\left.v\right|_{\partial B_{\eta}(0)}=0
\end{array}\right.
$$

We define $w(x, t)=(t-\tau) v(x)$ for $t \in(\tau, T)$, which satisfies

$$
\left\{\begin{array}{l}
w_{t}-\operatorname{div}\left(|x|^{-2 \gamma} \nabla w\right)=v(x)+(t-\tau) \frac{\bar{\varepsilon}}{|x|^{2(\gamma+1)}} \quad \text { in } B_{\eta}(0) \times(\tau, T), \\
\left.w\right|_{\partial B_{\eta}(0)}=0
\end{array}\right.
$$

and $\lim _{t \rightarrow \tau} \int_{B_{\eta}(0)} w(x, t) \phi(x) d x=0$ for all $\phi \in C_{0}^{\infty}\left(B_{\eta}(0)\right)$. Hence $w(x, t)$ is an energy solution and thus a weak solution to 31 . 
Since $w_{t}-\operatorname{div}\left(|x|^{-2 \gamma} \nabla w\right) \leq v(x)+T \bar{\varepsilon} /|x|^{2(\gamma+1)}$ and $\lim _{|x| \rightarrow 0}|x|^{2(\gamma+1)} v(x)=0$, one can choose $\bar{\varepsilon}$ and $\eta$ such that $w_{t}-\operatorname{div}\left(|x|^{-2 \gamma} \nabla w\right) \leq \varepsilon /|x|^{2(\gamma+1)}$ in $B_{\eta}(0) \times(\tau, T)$.

Let $w_{n}$ be the unique positive solution to the problem

$$
\left\{\begin{array}{l}
\left(w_{n}\right)_{t}-\operatorname{div}\left(|x|^{-2 \gamma} \nabla w_{n}\right)=\varepsilon a_{n}(x) \quad \text { in } B_{r}(0) \times(\tau, T), \\
\left.w_{n}\right|_{\partial B_{r}(0)}=0
\end{array}\right.
$$

Then by comparison,

$$
w(x, t) \leq w_{1}(x, t)=\lim _{n \rightarrow \infty} w_{n}(x, t) .
$$

Since $u_{n}(x, t) \geq u_{1}(x, t) \geq \varepsilon$, as above by the weak comparison principle, $u_{n}(x, t) \geq$ $w_{n}(x, t)$ for all $(x, t) \in B_{r}(0) \times(\tau, T)$. Taking into account that $\lim _{|x| \rightarrow 0} w(x, t)=\infty$ uniformly in $t \in\left[\tau_{1}, T_{1}\right] \subset(\tau, T)$, we obtain

$$
\lim _{|x| \rightarrow 0} u(x, t)=\lim _{|x| \rightarrow 0} \lim _{n \rightarrow \infty} u_{n}(x, t)=\infty \quad \forall t \in\left[\tau_{1}, T_{1}\right] .
$$

Then for all $c \gg 1$ there exist $n_{0} \in \mathbb{N}$ and $\eta_{1}>0$ such that for all $n \geq n_{0}, u_{n}(x, t) \geq c$ in $B_{\eta_{1}}(0)$ and uniformly in $t \in\left[\tau_{1}, T_{1}\right]$.

Let $B_{\bar{\eta}}(0) \subset \subset B_{\eta_{1}}(0)$ and consider $\psi \in C_{0}^{\infty}\left(B_{\bar{\eta}}(0)\right)$. By using Theorem 2.8, we get

$$
\int_{B_{\bar{\eta}}(0)}|\nabla \psi|^{2}|x|^{-2 \gamma} d x \geq \int_{B_{\bar{\eta}}(0)}|\psi|^{2} \frac{-\operatorname{div}\left(|x|^{-2 \gamma} \nabla u_{n}\right)}{u_{n}} d x \quad \text { for all } t \in\left[\tau_{1}, T_{1}\right] .
$$

Hence

$$
\int_{B_{\bar{\eta}}(0)}|\nabla \psi|^{2}|x|^{-2 \gamma} d x \geq \lambda \int_{B_{\bar{\eta}}(0)}|\psi|^{2} a_{n}(x) \frac{g_{n}(u)}{u_{n}} d x-\int_{B_{\bar{\eta}}(0)}|\psi|^{2} \frac{\left(u_{n}\right)_{t}}{u_{n}} d x
$$

and by integration we get

$$
\begin{aligned}
\int_{\tau_{1}}^{T_{1}} \int_{B_{\bar{\eta}}(0)} & |\nabla \psi|^{2}|x|^{-2 \gamma} d x d t \\
& \geq \lambda \int_{\tau_{1}}^{T_{1}} \int_{B_{\bar{\eta}}(0)}|\psi|^{2} a_{n}(x) \frac{g_{n}(u)}{u_{n}} d x d t-\int_{\tau_{1}}^{T_{1}} \int_{B_{\bar{\eta}}(0)}|\psi|^{2} \frac{\left(u_{n}\right)_{t}}{u_{n}} d x d t .
\end{aligned}
$$

We estimate directly the last integral on $\left(\tau_{1}, T_{1}\right)$ as follows:

$$
\begin{aligned}
& \left(T_{1}-\tau_{1}\right) \int_{B_{\bar{\eta}}(0)}|\nabla \psi|^{2}|x|^{-2 \gamma} d x d t \\
& \quad \geq \lambda \int_{\tau_{1}}^{T_{1}} \int_{B_{\bar{\eta}}(0)}|\psi|^{2} a_{n}(x) \frac{g_{n}(u)}{u} d x d t-\int_{B_{\bar{\eta}}(0)}|\psi|^{2} \log \left(u_{n}\left(x, T_{1}\right)\right) d x,
\end{aligned}
$$

because if $x \in B_{\bar{\eta}}(0)$ then $u_{n}\left(x, T_{1}\right) \gg 1$. As $g_{n}(u) a_{n}(x) \nearrow u /|x|^{2(\gamma+1)} \in L^{1}\left(B_{\bar{\eta}}(0)\right)$ and $|\psi|^{2} / u \in L^{\infty}\left(B_{\bar{\eta}}(0)\right)$, using the monotone convergence theorem we conclude that

$$
\lambda \int_{\tau_{1}}^{T_{1}} \int_{B_{\bar{\eta}}(0)}|\psi|^{2} a_{n}(x) \frac{g_{n}(u)}{u} d x d t \rightarrow \lambda\left(T_{1}-\tau_{1}\right) \int_{B_{\bar{\eta}}(0)} \frac{|\psi|^{2}}{|x|^{2(\gamma+1)}} d x .
$$


We set $\zeta(x)=\frac{1}{T_{1}-\tau_{1}} \log \left(u\left(x, T_{1}\right)\right)$. Since $u\left(\cdot, T_{1}\right) \in L^{1}\left(B_{\bar{\eta}}(0)\right)$ we see that $\zeta \in L^{p}\left(B_{\bar{\eta}}(0)\right)$ for all $p \geq 1$. In particular $\zeta \in L^{p}\left(B_{\bar{\eta}}(0)\right)$, where $p>(N-2 \gamma) / 2$. We set

$$
\lambda(\bar{\eta})=\inf _{\left\{\phi \in C_{0}^{\infty}\left(B_{\bar{\eta}}(0)\right): \phi \neq 0\right\}} \frac{\int_{B_{\bar{\eta}}(0)}|\nabla \phi|^{2} d x}{\int_{B_{\bar{\eta}}(0)} \zeta \phi^{2} d x} .
$$

Then $\lambda(\bar{\eta}) \rightarrow \infty$ as $\bar{\eta} \rightarrow 0$. Hence for all $\varepsilon>0$ we get the existence of $\eta_{\varepsilon}$ such that $\lambda\left(\eta_{\varepsilon}\right) \geq 1 / \varepsilon$. Let $\varepsilon$ be such that $\lambda /(1+\varepsilon)>\lambda_{N, \gamma}$. We set $\bar{\eta}=\eta_{\varepsilon}$. Then from (34) we obtain

$$
\int_{B_{\bar{\eta}}(0)}|\nabla \psi|^{2}|x|^{-2 \gamma} d x \geq \frac{\lambda}{1+\varepsilon} \int_{B_{\bar{\eta}}(0)} \frac{|\psi|^{2}}{|x|^{2(\gamma+1)}} d x .
$$

Since $\lambda /(1+\varepsilon)>\lambda_{N, \gamma}$, this contradicts the optimality of $\lambda_{N, \gamma}$.

Remark 4.2. It is clear that Theorem 4.1 holds even if the initial data $u_{0}$ belongs to $L^{1}$. By contradiction, assume that there exists a positive solution $u$ to problem $(26)$ with initial data $u_{0} \in L^{1}(\Omega)$; then $u$ is a supersolution to problem 26 with bounded initial data $u_{0, n}(x)=\min \left\{n, u_{0}(x)\right\}$ and this contradicts Theorem 4.1 for bounded data.

As a consequence we get the following corollaries.

Corollary 4.3. If $u_{0} \equiv 0$ then the unique nonnegative weak solution to problem (26) with $1+\gamma>0$ is $u \equiv 0$.

Corollary 4.4. Suppose $f \in L^{1}(\Omega \times(0, T))$ and $u_{0} \in L^{1}(\Omega)$ are positive functions such that $\left(f, u_{0}\right) \neq(0,0)$, and $1+\gamma>0$. Then for $\lambda>\lambda_{N, \gamma}$ the problem

$$
\left\{\begin{array}{l}
u_{t}-\operatorname{div}\left(|x|^{-2 \gamma} \nabla u\right)=\lambda \frac{u}{|x|^{2(\gamma+1)}}+f, \quad u \geq 0 \quad \text { in } \Omega \times(0, T), \\
u=0 \text { on } \partial \Omega \times(0, T), \\
u(x, 0)=u_{0}(x) \text { in } \Omega,
\end{array}\right.
$$

has no weak positive solution.

Notice that in the proof of Theorem 4.1 no explicit representation formula is needed, and so it is an alternative proof to the one given by Baras-Goldstein [BG] in the case $\gamma=0$. See also Cabré-Martel [CM].

Moreover we prove the following blow-up result.

Lemma 4.5. Assume that $1+\gamma>0$. Let $u_{n}$ be the solution to the problem

$$
\left\{\begin{array}{l}
\frac{\partial u_{n}}{\partial t}-\operatorname{div}\left(|x|^{-2 \gamma} \nabla u_{n}\right)=\lambda a_{n}(x) g_{n}\left(u_{n}\right) \quad \text { in } \Omega \times(0, T), \\
u_{n} \geq 0 \quad \text { in } \Omega \\
\left.u_{n}\right|_{\partial \Omega}=0, \quad u_{n}(x, 0)=u_{0}(x) \quad \text { in } \Omega,
\end{array}\right.
$$

where $a_{n}(x), g_{n}(s)$ are defined in (27), (28) and $u_{0} \in L^{\infty}(\Omega), u_{0} \geq 0$ and $u_{0} \not \equiv 0$. Then for all $r>0$ such that $B_{4 r}(0) \subset \Omega$ and all $t \in(0, T)$ we have

$$
\int_{B_{r}(0)} u_{n}(x, t) d x \rightarrow \infty \quad \text { as } n \rightarrow \infty .
$$


Proof. We argue by contradiction. Let $\tau, r_{0}>0$ be such that

$$
\int_{B_{r_{0}}(0)} u_{n}(x, \tau) d x \leq C_{0} \quad \text { for all } n .
$$

Notice that $\left\{u_{n}\right\}$ is a nondecreasing sequence. Thus there exists $u(x, \tau) \in L^{1}\left(B_{r_{0}}(0)\right)$ such that $u_{n}(x, \tau) \nearrow u(x, \tau)$ for all $x \in B_{r_{0}}(0)$. Without loss of generality we can assume that $B_{4 r_{0}}(0) \subset \Omega$. Then by the weak Harnack inequality for $u_{n}$, there exists $C_{1} \equiv C_{1}\left(\tau, N, \gamma, r_{0}\right)$ such that for all $t \in(\tau / 4, \tau / 3)$ we have

$$
\begin{aligned}
\int_{B_{r_{0}}(0)} u_{n}(x, t) d x & \leq C_{1} \inf \left\{u_{n}(x, t) \mid x \in B_{r_{0}}(0) \times(\tau / 2,3 \tau / 2)\right\} \\
& \leq C_{1} \frac{1}{\left|B_{r_{0}}(0)\right|} \int_{B_{r_{0}}(0)} u_{n}(x, \tau) d x \leq C_{2}
\end{aligned}
$$

for all $n \in \mathbb{N}$. Fix $\bar{\tau}$ such that $(\bar{\tau} / 5, \bar{\tau} / 4) \subset(\tau / 4, \tau / 3)$. Then we can define $u(x, t)$ for all $(x, t) \in B_{r_{0}}(0) \times(\bar{\tau} / 5, \bar{\tau} / 4)$ and $\int_{B_{r_{0}}(0)} u(x, t) d x<\infty$, uniformly in $t \in(\bar{\tau} / 5, \bar{\tau} / 4)$. Moreover, using Theorem 2.2, we get the existence of $\varepsilon_{1}>0$ such that $u_{n} \geq u_{1}>\varepsilon_{1}$ in $B_{r_{0}}(0) \times(\bar{\tau} / 5, \bar{\tau} / 4) \subset(0, T)$. Let

$$
\mu=\lambda \inf _{B_{r_{0}}(0) \times(\bar{\tau} / 5, \bar{\tau} / 4)} g_{1}\left(u_{1}(x, t)\right), \quad \varepsilon=\min \left\{\varepsilon_{1}, \mu\right\} .
$$

Since $u_{n}(x, t) \geq w_{n}(x, t)$, where $w_{n}$ is the solution to the problem

$$
\left\{\begin{array}{l}
\left(w_{n}\right)_{t}-\operatorname{div}\left(|x|^{-2 \gamma} \nabla w_{n}\right)=\varepsilon a_{n}(x) \quad \text { in } B_{r_{0}}(0) \times(\bar{\tau} / 5, \bar{\tau} / 4), \\
\left.w_{n}\right|_{\partial B_{r_{0}}(0)}=0, \quad w_{n}(x, \bar{\tau} / 5)=\varepsilon \quad \text { in } B_{r_{0}}(0),
\end{array}\right.
$$

using the facts that $w_{n} \uparrow w$ and $\lim _{|x| \rightarrow 0} w(x, t)=\infty$ uniformly in $\left(\tau_{2}, \tau_{3}\right)$, where $\bar{\tau} / 5<\tau_{2}<\tau_{3}<\bar{\tau} / 4$ (see the proof of Theorem 4.1), we conclude that

$$
\lim _{n \rightarrow \infty} \lim _{|x| \rightarrow 0} u_{n}(x, t)=\infty \quad \text { uniformly in }\left(\tau_{2}, \tau_{3}\right) .
$$

Let $B_{\bar{\eta}}(0) \subset \subset \Omega$ be such that $u_{n}(x, t) \gg 1$ for all $x \in B_{\bar{\eta}}(0) \times\left(t_{1}, t_{2}\right)$ with $\tau_{2}<t_{1}<$ $t_{2}<\tau_{3}$, and $\psi \in C_{0}^{\infty}\left(B_{\bar{\eta}}(0)\right)$. Using Theorem 2.8 we obtain

$$
\int_{B_{\bar{\eta}}(0)}|\nabla \psi|^{2}|x|^{-2 \gamma} d x \geq \lambda \int_{B_{\bar{\eta}}(0)}|\psi|^{2} a_{n}(x) \frac{g_{n}\left(u_{n}\right)}{u_{n}} d x-\int_{B_{\bar{\eta}}(0)}|\psi|^{2} \frac{\left(u_{n}\right)_{t}}{u_{n}} d x .
$$

By integration we get

$$
\begin{aligned}
\int_{t_{1}}^{t_{2}} \int_{B_{\bar{\eta}}(0)} & |\nabla \psi|^{2}|x|^{-2 \gamma} d x d t \\
& \geq \lambda \int_{t_{1}}^{t_{2}} \int_{B_{\bar{\eta}}(0)}|\psi|^{2} a_{n}(x) \frac{g_{n}\left(u_{n}\right)}{u_{n}} d x d t-\int_{t_{1}}^{t_{2}} \int_{B_{\bar{\eta}}(0)}|\psi|^{2} \frac{\left(u_{n}\right)_{t}}{u_{n}} d x d t .
\end{aligned}
$$

Since $u_{n} \uparrow u$ in $L^{1}\left(B_{\bar{\eta}}(0) \times\left(t_{1}, t_{2}\right)\right)$, using the same argument as in the proof of Theorem 4.1 we get

$$
\int_{B_{\bar{\eta}}(0)}|\nabla \psi|^{2}|x|^{-2 \gamma} d x \geq \frac{\lambda}{1+\varepsilon} \int_{B_{\bar{\eta}}(0)} \frac{|\psi|^{2}}{|x|^{2(\gamma+1)}} d x, \quad \forall \psi \in C_{0}^{\infty}\left(B_{\bar{\eta}}(0)\right),
$$


where $\varepsilon$ can be chosen such that $\lambda /(1+\varepsilon)>\lambda_{N, \gamma}$. This contradicts the optimality of $\lambda_{N, \gamma}$.

We are now able to formulate the main blow-up result.

Theorem 4.6. Let $u_{n}$ be the positive solution to problem with $1+\gamma>0$. Then for all $\left(x_{0}, t_{0}\right) \in \Omega \times(0, T)$ we have $\lim _{n \rightarrow \infty} u_{n}\left(x_{0}, t_{0}\right)=\infty$.

Proof. Let $\left(x_{0}, t_{0}\right) \in \Omega \times(0, T)$. If $x_{0} \in B_{r}(0)$ and $B_{4 r}(0) \subset \Omega$, then using the Harnack inequality we get

$$
u_{n}\left(x_{0}, t_{0}\right) \geq c \int_{B_{r}(0)} u_{n}\left(x, t_{0}-\varepsilon\right) d x,
$$

where $c=c(N, \gamma, r)$ and $\varepsilon>0$ is such that $t_{0}-\varepsilon>0$. By Lemma 4.5 we find that $\int_{B_{r}(0)} u_{n}\left(x, t_{0}-\varepsilon\right) d x \rightarrow \infty$, hence

$$
\lim _{n \rightarrow \infty} u_{n}\left(x_{0}, t_{0}\right)=\infty
$$

If $x_{0} \notin B_{r}(0)$, we first suppose that $B_{r}(0) \cap B_{r}\left(x_{0}\right) \neq \emptyset$. Then there exists $y \in \Omega$ such that $B_{\eta}(y) \subset B_{r}(0) \cap B_{r}\left(x_{0}\right)$. Therefore using again the Harnack inequality and Lemma 4.5 we obtain

$$
\begin{aligned}
u_{n}\left(x_{0}, t_{0}\right) & \geq c \int_{B_{r}\left(x_{0}\right)} u_{n}\left(x, t_{0}-\varepsilon\right) d x \geq c \int_{\bar{B}_{\eta}(y)} u_{n}\left(x, t_{0}-\varepsilon\right) d x \\
& \geq c \inf _{x \in \bar{B}_{\eta}(y)} u_{n}\left(x, t_{0}-\varepsilon\right) \geq c u_{n}\left(x_{1}, t_{0}-\varepsilon\right),
\end{aligned}
$$

where $c$ is a positive constant independent of $u_{n}$. Since $x_{1} \in B_{r}(0)$, we have $u_{n}\left(x_{1}, t_{0}-\varepsilon\right)$ $\rightarrow \infty$, and we find that $u_{n}\left(x_{0}, t_{0}\right) \rightarrow \infty$ for $n \rightarrow \infty$. In the general case, if $x_{0} \in \Omega$ is arbitrary, then since $d\left(x, B_{r}(0)\right)<\infty$, using an iteration argument, we conclude in a finite number of steps.

4.2. The superlinear case: $\alpha>1$ with $1+\gamma>0$

In this subsection we deal with the following problem:

$$
\left\{\begin{array}{l}
u_{t}-\operatorname{div}\left(|x|^{-2 \gamma} \nabla u\right)=\frac{u^{\alpha}}{|x|^{2(\gamma+1)}} \quad \text { in } \Omega \times(0, T) \\
u=0 \text { on } \partial \Omega \times(0, T) \\
u(x, 0)=u_{0}(x) \quad \text { in } \Omega
\end{array}\right.
$$

where $\alpha>1$ and $1+\gamma>0$. Here the behavior of the problem is quite different in comparison to the linear case, in particular it is independent of $\lambda$. So we formulate the result with $\lambda=1$.

Theorem 4.7. Let $u_{0}$ be a positive function such that $u_{0} \in L^{\infty}(\Omega)$ and $u_{0} \not \equiv 0$. Then problem (41) with $1+\gamma>0$ has no weak positive solution. If $u_{0} \equiv 0$ then the unique nonnegative solution is $u \equiv 0$. 
Proof. Assume that (41) has a positive weak solution $u$. Let $a_{n}$ be defined in (27). Define $g_{n}(s)=\min \left\{n, s^{\alpha}\right\}$ and $f_{n}(x, t)=a_{n}(x) g_{n}(u(x, t))$; then $f_{n} \in L^{\infty}(\Omega \times(0, T))$ and $f_{n}(x, t) \quad \nearrow f(x, t)=u(x, t)^{\alpha} /|x|^{2(\gamma+1)}$ in $L^{1}(\Omega \times(0, T))$. Since $|x|^{2 \gamma} f_{n} \in$ $L^{r}\left(\Omega,|x|^{-2 \gamma} d x\right)$ with $r>(N-2 \gamma) / 2$, we have the required integrability to obtain $L^{\infty}$ estimates. (See $[\overline{\mathrm{ChF}}]$ and $[\overline{\mathrm{AP}}]$.) Consider the unique positive global solution $u_{n}$ to the problem

$$
\left\{\begin{array}{l}
\left(u_{n}\right)_{t}-\operatorname{div}\left(|x|^{-2 \gamma} \nabla u_{n}\right)=f_{n}(x, t) \quad \text { in } \Omega \times(0, T) \\
u_{n}=0 \quad \text { on } \partial \Omega \times(0, T) \\
u_{n}(x, 0)=u_{0}(x) \quad \text { in } \Omega
\end{array}\right.
$$

Then $u_{n} \in L^{\infty}(\Omega \times(0, T))$, and since $u_{0} \not \equiv 0$, the weak Harnack inequality implies that $u_{n}>0$ in $\Omega \times(0, T)$. Since $\left\{f_{n}\right\}$ is increasing, $\left\{u_{n}\right\}$ is also increasing and converges to $u$ in $C\left([0, T] ; L^{1}(\Omega)\right)$. Again the Harnack inequality gives

$$
u \geq u_{1} \geq \varepsilon \quad \text { a.e. in } B_{\eta}(0) \times(\tau, T)
$$

for some $0<\tau<T$ and $B_{\eta}(0) \subset \subset \Omega$. By the same arguments as in the proof of Theorem 4.1 we find that

$$
\lim _{|x| \rightarrow 0} u(x, t)=\lim _{|x| \rightarrow 0} \lim _{n \rightarrow \infty} u_{n}(x, t)=\infty \quad \text { uniformly in } t \in(\tau, T) .
$$

Then for all $c>0$ there exist $n_{0} \in \mathbb{N}$ and $\eta_{1}>0$ such that $u_{n}(x, t) \geq c$ for all $x \in B_{\eta_{1}}(0)$ and all $t \in(\tau, T)$. We choose $\bar{c}>\sup \{1, \alpha-1\}$ such that

$$
(\alpha-1) \bar{c}^{\alpha-1}-4(\alpha-1) \frac{\log (\bar{c})}{T-\tau}>\lambda_{N, \gamma},
$$

where $\lambda_{N, \gamma}$ is the optimal constant in the Hardy inequality given in Proposition 2.5 Moreover there exist $\eta, 0<\eta \ll 1$, and $n_{0} \in \mathbb{N}$ such that if $n \geq n_{0}$ then $u_{n} \geq 2 \bar{c}$ in $B_{\eta}(0) \times(\tau, T)$.

Next we use arguments as in [BC]. Define

$$
\phi(s)= \begin{cases}\frac{1}{\alpha-1}\left(\frac{1}{\bar{c}^{\alpha-1}}-\frac{1}{s^{\alpha-1}}\right) & \text { if } s>\bar{c}, \\ \frac{1}{\bar{c}^{\alpha}}(s-\bar{c}) & \text { if } s \leq \bar{c} .\end{cases}
$$

Notice that $\phi \in C^{1}(\mathbb{R})$ is a concave function and $\phi^{\prime}(s)=1 / s^{\alpha}$ for all $s>\bar{c}$, in particular $0<\phi^{\prime}(s)<1 / \bar{c}^{\alpha}$. Since $\phi(s)$ is a regular function for $s>\bar{c}$, and $u_{n}>\bar{c}$ for all $n \geq n_{0}$, it follows that

$$
-\operatorname{div}\left(|x|^{-2 \gamma} \nabla \phi\left(u_{n}\right)\right)=\phi^{\prime}\left(u_{n}\right)\left(-\operatorname{div}\left(|x|^{-2 \gamma} \nabla u_{n}\right)\right)-\phi^{\prime \prime}\left(u_{n}\right)|x|^{-2 \gamma}\left|\nabla u_{n}\right|^{2} .
$$

It is clear that $\phi\left(u_{n}\right) \leq \frac{1}{\alpha-1} \frac{1}{\bar{c}^{\alpha-1}}$. Since $\phi^{\prime \prime}<0$ and $u_{n}(x)>\bar{c}>1$ in $B_{\eta}(0)$, we obtain

$$
\left(\phi\left(u_{n}\right)\right)_{t}-\operatorname{div}\left(|x|^{-2 \gamma} \nabla \phi\left(u_{n}\right)\right) \geq \phi^{\prime}\left(u_{n}\right)\left(\left(u_{n}\right)_{t}-\operatorname{div}\left(|x|^{-2 \gamma} \nabla u_{n}\right)\right)=\phi^{\prime}\left(u_{n}\right) f_{n} .
$$

Consider $\psi \in C_{0}^{\infty}\left(B_{\eta}(0)\right)$. Using Theorem 2.8, we get

$$
\int_{B_{\eta}(0)}|\nabla \psi|^{2}|x|^{-2 \gamma} d x \geq \int_{B_{\eta}(0)}|\psi|^{2} \frac{-\operatorname{div}\left(|x|^{-2 \gamma} \nabla \phi\left(u_{n}\right)\right)}{\phi\left(u_{n}\right)} d x,
$$


hence

$$
\begin{aligned}
\int_{B_{\eta}(0)}|\nabla \psi|^{2}|x|^{-2 \gamma} & d x \\
& \geq \int_{B_{\eta}(0)}|\psi|^{2} f_{n} \frac{\phi^{\prime}\left(u_{n}\right)}{\phi\left(u_{n}\right)} d x-\int_{B_{\eta}(0)}|\psi|^{2} \frac{\left(u_{n}\right)_{t} \phi^{\prime}\left(u_{n}\right)}{\phi\left(u_{n}\right)} d x .
\end{aligned}
$$

By integration over $(\tau, T)$ we obtain

$$
\begin{aligned}
& \int_{\tau}^{T} \int_{B_{\eta}(0)}|\nabla \psi|^{2}|x|^{-2 \gamma} d x d t \\
& \quad \geq \int_{\tau}^{T} \int_{B_{\eta}(0)}|\psi|^{2} f_{n} \frac{\phi^{\prime}\left(u_{n}\right)}{\phi\left(u_{n}\right)} d x d t-\int_{\tau}^{T} \int_{B_{\eta}(0)}|\psi|^{2} \frac{\left(u_{n}\right)_{t} \phi^{\prime}\left(u_{n}\right)}{\phi\left(u_{n}\right)} d x d t .
\end{aligned}
$$

Since $x \in B_{\eta}(0)$, we have $u_{n}(x, t)>2 \bar{c}$. Therefore

$$
\frac{1}{\phi\left(u_{n}\right)} \geq(\alpha-1) \bar{c}^{\alpha-1} \text {. }
$$

On the other hand, $\phi^{\prime}\left(u_{n}\right) f_{n} \rightarrow \phi^{\prime}(u) u^{\alpha} /|x|^{2(\gamma+1)}$ a.e. in $\Omega$. Since $\phi^{\prime}\left(u_{n}\right) \leq a$ for all $n \geq n_{0}$ and $x \in B_{\eta}(0)$, we obtain $\phi^{\prime}\left(u_{n}\right) f_{n} \leq a f$. Hence by the dominated convergence theorem we conclude that

$$
\phi^{\prime}\left(u_{n}\right) f_{n} \rightarrow \phi^{\prime}(u) \frac{u^{\alpha}}{|x|^{2(\gamma+1)}}=\frac{1}{|x|^{2(\gamma+1)}} \quad \text { in } L^{1}\left(B_{\eta}(0) \times(\tau, T)\right) .
$$

Therefore

$$
\int_{\tau}^{T} \int_{B_{\bar{\eta}}(0)}|\psi|^{2} f_{n} \frac{\phi^{\prime}\left(u_{n}\right)}{\phi\left(u_{n}\right)} d x d t \geq(T-\tau)(\alpha-1) \bar{c}^{\alpha-1} \int_{B_{\bar{\eta}}(0)} \frac{|\psi|^{2}}{|x|^{2(\gamma+1)}} d x+o(1) .
$$

To estimate the term involving the $t$ derivative, we proceed as follows. Let

$$
I=\left.\left|\int_{B_{\bar{\eta}}(0)}\right| \psi\right|^{2} \int_{\tau}^{T} \frac{\left(u_{n}\right)_{t} \phi^{\prime}\left(u_{n}\right)}{\phi\left(u_{n}\right)} d t d x|=| \int_{B_{\bar{\eta}}(0)}|\psi|^{2} \log \left(\frac{\phi\left(u_{n}(T)\right)}{\phi\left(u_{n}(\tau)\right)}\right) d x \mid .
$$

Since $\phi\left(u_{n}\right) \leq \phi(u)$ we get

$$
I \leq 2 \int_{B_{\bar{\eta}}(0)}|\psi|^{2}|\log \phi(u(T))| d x \leq 4(\alpha-1) \log (\bar{c}) \int_{B_{\bar{\eta}}(0)}|\psi|^{2} d x .
$$

Hence, by substitution in 46 and integrating in time, we obtain

$$
\begin{aligned}
\int_{B_{\bar{\eta}}(0)}|\nabla \psi|^{2}|x|^{-2 \gamma} d x & \geq\left((\alpha-1) \bar{c}^{\alpha-1}-4(\alpha-1) \frac{\log (\bar{c})}{T-\tau}\right) \int_{B_{\bar{\eta}}(0)} \frac{|\psi|^{p}}{|x|^{2(\gamma+1)}} d x \\
& =\Lambda \int_{B_{\bar{\eta}}(0)} \frac{|\psi|^{2}}{|x|^{2(\gamma+1)}} d x,
\end{aligned}
$$

where

$$
\Lambda:=(\alpha-1) \bar{c}^{\alpha-1}-4(\alpha-1) \frac{\log (\bar{c})}{T-\tau}>\lambda_{N, \gamma}
$$

by 43 , which contradicts the optimality of $\lambda_{N, \gamma}$. 
Corollary 4.8. Suppose $f \in L^{1}(\Omega \times(0, T))$ and $u_{0} \in L^{1}(\Omega)$ are positive functions such that $\left(f, u_{0}\right) \neq(0,0)$ and $1+\gamma>0$. Then the problem

$$
\left\{\begin{array}{l}
u_{t}-\operatorname{div}\left(|x|^{-2 \gamma} \nabla u\right)=\frac{u^{\alpha}}{|x|^{2(\gamma+1)}}+f, \quad u \geq 0 \quad \text { in } \Omega \times(0, T), \\
u=0 \quad \text { on } \partial \Omega \times(0, T), \\
u(x, 0)=u_{0}(x) \quad \text { in } \Omega,
\end{array}\right.
$$

has no positive weak solution.

As a consequence we have the following blow-up result.

Lemma 4.9. Let $u_{n}$ be the solution to the problem

$$
\left\{\begin{array}{l}
\frac{\partial u_{n}}{\partial t}-\operatorname{div}\left(|x|^{-2 \gamma} \nabla u_{n}\right)=a_{n}(x) g_{n}\left(u_{n}\right) \quad \text { in } \Omega \times(0, T), \\
u_{n} \geq 0 \quad \text { in } \Omega,\left.\quad u_{n}\right|_{\partial \Omega}=0 \\
u_{n}(0, x)=u_{0}(x) \quad \text { in } \Omega .
\end{array}\right.
$$

Then for all $r>0$ such that $B_{4 r}(0) \subset \Omega$, and for all $t \in(0, T)$,

$$
\int_{B_{r}(0)} u_{n}(x, t) d x \rightarrow \infty \quad \text { as } n \rightarrow \infty .
$$

Proof. We proceed as in the proof of Lemma 4.5 Assume the existence of $\tau, r_{0}>0$ such that

$$
\int_{B_{r_{0}}(0)} u_{n}(x, \tau) d x \leq C \quad \text { for all } n .
$$

Since $u_{n}$ are increasing, there exists $u(x, \tau) \in L^{1}\left(B_{r_{0}}(0)\right)$ such that

$$
u_{n}(x, \tau) \nearrow u(x, \tau) \text { for all } x \in B_{r_{0}}(0) .
$$

Without loss of generality we can assume that $B_{4 r_{0}}(0) \subset \Omega$. Then using the weak Harnack inequality as in the proof of Lemma 4.5 we get the existence of $\tau>0$ such that $\int_{B_{r_{0}}(0)} u_{n}(x, t) d x \leq C\left(r_{0}, \tau\right)$ for all $0<\tau / 2 \leq t<\tau$. Then we obtain $u(\cdot, t) \in$ $L^{1}\left(B_{r_{0}}(0)\right)$ for all $t \in\left(\tau_{1} / 4, \tau_{1}\right) \subset(\tau / 2, \tau)$ and $\int_{B_{r_{0}}(0)} u(x, t) d x<C$, where $C$ is independent of $t$. Moreover as in Lemma 4.5 one can prove that

$$
\lim _{|x| \rightarrow 0} \lim _{n \rightarrow \infty} u_{n}(x, t)=\infty \quad \text { uniformly for all } t \in\left(\tau_{2}, \tau_{3}\right),
$$

where $\tau_{1} / 4<\tau_{2}<\tau_{3}<\tau_{1}$. Fix $B_{r_{0}}(0) \subset \subset \Omega$ such that $u_{n} \gg \bar{c}$ in $B_{r_{0}}(0) \times\left(\tau_{2}, \tau_{3}\right)$. Let $\psi \in C_{0}^{\infty}\left(B_{r_{0}}(0)\right)$. By Theorem 2.8 we get

$$
\int_{B_{r_{0}}(0)}|\nabla \psi|^{2}|x|^{-2 \gamma} d x \geq \int_{B_{r_{0}}(0)} \frac{-\operatorname{div}\left(|x|^{-2 \gamma} \nabla u_{n}\right)}{u_{n}} \psi^{2} d x .
$$

Therefore as in the proof of Theorem 4.7 we obtain

$$
\int_{B_{\bar{\eta}}(0)}|\nabla \psi|^{2}|x|^{-2 \gamma} d x \geq\left((\alpha-1) \bar{c}^{\alpha-1}-4(\alpha-1) \frac{\log (\bar{c})}{\tau_{3}-\tau_{2}}\right) \int_{B_{\bar{\eta}}(0)} \frac{|\psi|^{p}}{|x|^{2(\gamma+1)}} d x
$$


for all $\psi \in C_{0}^{\infty}\left(B_{\bar{\eta}}(0)\right)$. Since we can choose $\bar{c}$ with $(\alpha-1) \bar{c}^{\alpha-1}-4(\alpha-1) \frac{\log (\bar{c})}{\tau_{3}-\tau_{2}}>\lambda_{N, \gamma}$, we reach a contradiction with the optimality of $\lambda_{N, \gamma}$.

As a consequence of the weak Harnack inequality we obtain the main blow-up result.

Theorem 4.10. Assume that $u_{n}$ is the positive solution to problem (48) with $1+\gamma>0$. Then for all $\left(x_{0}, t_{0}\right) \in \Omega \times(0, T)$ we have $\lim _{n \rightarrow \infty} u_{n}\left(x_{0}, t_{0}\right)=\infty$.

Remark 4.11. Notice that if we consider the problem

$$
\left\{\begin{array}{l}
u_{t}-\operatorname{div}\left(|x|^{-2 \gamma} \nabla u\right)=\lambda \frac{u}{|x|^{2(\gamma+1)}}+f \quad \text { in } \Omega \times(0, T), \\
u \geq 0 \quad \text { in } \Omega \times(0, T), \quad u=0 \quad \text { on } \partial \Omega \times(0, T) \\
u(x, 0)=u_{0}(x) \text { in } \Omega,
\end{array}\right.
$$

with a nonnegative function $f$ such that $f(x, t)>c>0$ in a neighborhood of $\{0\} \times(0, T)$ and under the general hypothesis on $\Omega, \lambda>\lambda_{N, \gamma}, u_{0} \geq 0$, then we can repeat the proof of the nonexistence and blow-up results without the restriction $-\infty<\gamma<(N-2) / 2$. The relevant property is that in this case the equation

$$
u_{t}-\operatorname{div}\left(|x|^{-2 \gamma} \nabla u\right)=f
$$

satisfies the strong maximum principle.

\section{Existence and nonuniqueness results for the sublinear case: $0<\alpha<1$}

In this section we study the sublinear case, $0<\alpha<1$.

Theorem 5.1. Let $u_{0} \in L^{2}(\Omega)$ be a positive function. Assume that $0<\alpha<1$. Then the problem

$$
\left\{\begin{array}{l}
u_{t}-\operatorname{div}\left(|x|^{-2 \gamma} \nabla u\right)=\frac{u^{\alpha}}{|x|^{2(\gamma+1)}}, \quad u \geq 0 \quad \text { in } \Omega \times(0, T), \\
u=0 \text { on } \partial \Omega \times(0, T) \\
u(x, 0)=u_{0}(x) \quad \text { in } \Omega,
\end{array}\right.
$$

has a global solution $u \in L^{2}\left((0, T) ; \mathcal{D}_{0, \gamma}^{1,2}(\Omega)\right) \cap C\left([0, T] ; L^{2}(\Omega)\right)$.

Proof. The existence of solution for $t$ small can be easily proved. Moreover using $u$ as a test function in 51) we obtain

$$
\int_{\Omega} u(x, T)^{2} d x+\int_{0}^{T} \int_{\Omega}|\nabla u|^{2}|x|^{-2 \gamma} d x d t=\int_{\Omega} u_{0}^{2} d x+\int_{0}^{T} \int_{\Omega} \frac{u^{\alpha+1}}{|x|^{2(\gamma+1)}} d x d t .
$$

By the Hölder and Young inequalities we get

$$
\begin{aligned}
\int_{\Omega} u_{n}(x, T)^{2} d x+\int_{0}^{T} \int_{\Omega}\left|\nabla u_{n}\right|^{2}|x|^{-2 \gamma} d x d t \\
\leq \int_{\Omega} u_{0}^{2} d x+\int_{0}^{T}\left(\int_{\Omega} \frac{d x}{|x|^{2(\gamma+1)}}\right)^{(1-\alpha) / 2}\left(\int_{\Omega} \frac{u^{2} d x}{|x|^{2(\gamma+1)}}\right)^{(\alpha+1) / 2} d t \\
\leq \int_{\Omega} u_{0}^{2} d x+\frac{\alpha+1}{2} \varepsilon^{(\alpha+1) / 2} \int_{0}^{T} \int_{\Omega} \frac{u^{2}}{|x|^{2(\gamma+1)}} d x d t+c \varepsilon^{-(1-\alpha) / 2} T
\end{aligned}
$$


By Proposition 2.5 we obtain

$$
\begin{aligned}
\int_{\Omega} u_{n}(x, T)^{2} d x+\left(1-\lambda_{N, \gamma}^{-1} \frac{\alpha+1}{2} \varepsilon^{(\alpha+1) / 2}\right) \int_{0}^{T} & \int_{\Omega}\left|\nabla u_{n}\right|^{2}|x|^{-2 \gamma} d x d t \\
& \leq c \varepsilon^{-(1-\alpha) / 2} T+\int_{\Omega} u_{0}^{2} d x
\end{aligned}
$$

and choosing $\varepsilon$ small enough we conclude that the solution is defined in $[0, T]$ for arbitrary $T$.

Consider now the stationary problem

$$
-\operatorname{div}\left(|x|^{-2 \gamma} \nabla w\right)=\frac{w^{\alpha}}{|x|^{2(\gamma+1)}},\left.\quad w\right|_{\partial \Omega}=0 .
$$

An elementary modification of the arguments in [AP2] allows us to prove that there exists a unique solution $w \geq 0$ to [52], which is the stationary solution to problem (51) with $u_{0} \equiv w$.

Since $\alpha<1$, using a construction by H. Fujita in $[\bar{F}]$ for the heat equation, we obtain the following nonuniqueness result.

Theorem 5.2. Let $w$ be the unique positive solution to problem [52). Then the problem

$$
\left\{\begin{array}{l}
u_{t}-\operatorname{div}\left(|x|^{-2 \gamma} \nabla u\right)=\frac{u^{\alpha}}{|x|^{2(\gamma+1)}}, \quad u \geq 0 \quad \text { in } \Omega \times(0, T), \\
u=0 \text { on } \partial \Omega \times(0, T) \\
u(x, 0)=0 \text { in } \Omega
\end{array}\right.
$$

has a positive maximal solution $u \neq \equiv$, and moreover $u \nearrow w$ in $\mathcal{D}_{0, \gamma}^{1,2}(\Omega)$ as $t \rightarrow \infty$.

To prove the above theorem we will use the following result.

Lemma 5.3. Let $v \in L^{2}\left((0, T) ; \mathcal{D}_{0, \gamma}^{1,2}(\Omega)\right) \cap C\left([0, T] ; L^{1}(\Omega)\right)$ be a positive subsolution to problem 53. Then $\frac{d}{d t} \int_{\Omega}(v-w)_{+}^{2} d x \leq 0$, that is,

$$
\int_{\Omega}(v-w)_{+}^{2} d x \leq \int_{\Omega}(v(0)-w)_{+}^{2} d x \quad \text { for all } t \geq 0 .
$$

Proof. Since $v$ is a subsolution we get

$$
\left(v_{t}-w_{t}\right)-\operatorname{div}\left(|x|^{-2 \gamma} \nabla(v-w)\right) \leq \frac{v^{\alpha}-w^{\alpha}}{|x|^{2(\gamma+1)}} .
$$

Using $(v-w)_{+}$as a test function we obtain

$$
\begin{aligned}
\frac{d}{d t} \int_{\Omega}(v-w)_{+}^{2} d x+\int_{\Omega}\left|\nabla(v-w)_{+}\right|^{2}|x|^{-2 \gamma} d x & \leq \int_{\Omega} \frac{\left(v^{\alpha}-w^{\alpha}\right)(v-w)_{+}}{|x|^{2(\gamma+1)}} d x \\
& \leq \alpha \int_{\Omega} w^{\alpha-1} \frac{(v-w)_{+}^{2}}{|x|^{2(\gamma+1)}} d x .
\end{aligned}
$$


By Theorem 2.8 we get

$$
\begin{aligned}
\int_{\Omega}\left|\nabla(v-w)_{+}\right|^{2}|x|^{-2 \gamma} d x & \geq \int_{\Omega} \frac{-\operatorname{div}\left(|x|^{-2 \gamma} \nabla w\right)}{w}(v-w)_{+}^{2} d x \\
& \geq \int_{\Omega} \frac{w^{\alpha-1}}{|x|^{2(\gamma+1)}}(v-w)_{+}^{2} d x .
\end{aligned}
$$

Hence we conclude that $\frac{d}{d t} \int_{\Omega}(v-w)_{+}^{2} d x \leq 0$.

Proof of Theorem 5.2 Let $w$ be the unique positive solution to problem (52). Since $u_{0} \equiv 0$, it follows that $w$ is a supersolution to problem $\sqrt[53]{ }$. We set $u_{(0)}=w$ and we define $u_{n+1}$ as the unique solution to the problem

$$
\left\{\begin{array}{l}
\frac{\partial u_{n+1}}{\partial t}-\operatorname{div}\left(|x|^{-2 \gamma} \nabla u_{n+1}\right)=\frac{u_{n}^{\alpha}}{|x|^{2(\gamma+1)}}, \quad u_{n+1} \geq 0 \quad \text { in } \Omega \times\left(0, t_{0}\right), \\
u_{n+1}=0 \text { on } \partial \Omega \times\left(0, t_{0}\right), \\
u_{n+1}(x, 0)=0 \text { in } \Omega .
\end{array}\right.
$$

Since $\left\{u_{n}\right\}$ is a decreasing sequence and $u_{n} \leq u_{0}$ for all $n$, we get the existence of a solution $u$ to problem (53). We just have to prove that $u \neq 0$. We define $w_{\varepsilon}(x, t)=$ $\varepsilon^{1-\alpha}((1-\alpha) t)^{1-\alpha} \phi$, where $\varepsilon>0$ will be chosen later and $\phi$ is the first eigenfunction of the problem

$$
-\operatorname{div}\left(|x|^{-2 \gamma} \nabla \phi\right)=\lambda_{1} \frac{\phi}{|x|^{2 \gamma}},\left.\quad \phi\right|_{\partial \Omega}=0,
$$

such that $\|\phi\|_{L^{\infty}}=1$. Then we get

$$
\left\{\begin{array}{l}
\frac{\partial w_{\varepsilon}}{\partial t}-\operatorname{div}\left(|x|^{-2 \gamma} \nabla w_{\varepsilon}\right)=\left(\varepsilon^{\alpha+1} t+\varepsilon^{1-\alpha}((1-\alpha) t)^{1-\alpha}\right) \phi, \\
w_{\varepsilon} \geq 0 \text { in } \Omega \times\left(0, t_{0}\right), \quad w_{\varepsilon}=0 \quad \text { on } \partial \Omega \times\left(0, t_{0}\right), \quad w_{\varepsilon}(x, 0)=0 \quad \text { in } \Omega .
\end{array}\right.
$$

Choosing $\varepsilon$ such that $w_{\varepsilon} \leq w$, we can prove by induction that $u_{n} \geq w_{\varepsilon}$ for all $n$. Thus we conclude that $u \geq w_{\varepsilon}>0$.

Let $v$ be another solution to 53 . Then using Lemma 5.3 we conclude that $v \leq w=$ $u_{(0)}$, and using an iteration argument one can prove that $u_{n} \geq v$ for all $n$. Hence $u \geq v$ and the maximality of $u$ follows.

Let $s>0$. Then one can prove that $u(x, t+s) \geq u(x, t)$ for all $(x, t) \in \Omega \times(0, T)$, hence $u$ is increasing with respect to $t$ for all $x \in \Omega$. Taking $u$ as a test function in (53) and using the increasing property, we get

$$
\int_{\Omega}|\nabla u(x, t)|^{2}|x|^{-2 \gamma} d x \leq \int_{\Omega} \frac{u^{\alpha}}{|x|^{2(\gamma+1)}} d x .
$$

By the Hölder and Hardy-Sobolev inequalities (see (19), we deduce that for all $t>0$,

$$
\int_{\Omega}|\nabla u(x, t)|^{2}|x|^{-2 \gamma} d x \leq C(N, \gamma) .
$$

Hence we get the existence of a subsequence $t_{k} \uparrow \infty$ and $\bar{w} \in \mathcal{D}_{0, \gamma}^{1,2}(\Omega)$ such that $u\left(\cdot, t_{k}\right) \rightarrow \bar{w}$ as $t_{k} \rightarrow \infty$ and $u\left(\cdot, t_{k}\right) \rightarrow \bar{w}$ a.e. in $\Omega$ and in $L^{r}\left(|x|^{-2 \gamma} d x, \Omega\right)$ for all 
$1 \leq r<2_{\gamma}^{*}$. Since $u$ is increasing in $t$, we get the convergence for all subsequences. Then one can prove easily that $\bar{w}$ is a weak solution to problem (52), and as a consequence $w=\bar{w}$.

In the case $0 \leq u(x, 0)=u_{0} \leq w$, we can prove the following result.

Lemma 5.4. Let $u$ be a solution to problem (51) with $0 \leq u(x, 0) \leq w$. Then

$$
\lim _{t \rightarrow \infty} u(x, t)=w(x) \quad \text { a.e. }
$$

Proof. By Lemma 5.3 $u(x, t) \leq w(x)$ for all $(x, t) \in \Omega \times(0, \infty)$. Using $w(x)$ as a test function in problem (51) and taking into account that $0<\alpha<1, u^{\alpha} w \geq u w^{\alpha}$, we obtain

$$
\frac{d}{d t} \int_{\Omega} u(x, t) w(x) d x+\int_{\Omega} \frac{u w^{\alpha}}{|x|^{2(\gamma+1)}} d x=\int_{\Omega} \frac{u^{\alpha} w}{|x|^{2(\gamma+1)}} w d x \geq \int_{\Omega} \frac{u w^{\alpha}}{|x|^{2(\gamma+1)}} d x .
$$

Then

$$
\int_{\Omega} u(x, t) w(x) d x \geq \int_{\Omega} u(x, 0) w(x) d x>0 .
$$

As a consequence $u(x, t) \nrightarrow \rightarrow 0$ as $t \rightarrow \infty$. Since $0 \leq u(x, t) \leq w(x)$, it follows that $u(x, t) \rightarrow w_{1}(x)$, a solution to the stationary problem as $t \rightarrow \infty$. Then by uniqueness, $w_{1} \equiv w$.

Let $\psi(x) \equiv|x|^{-\kappa_{c}}$, where $\lambda<c<\lambda_{N, \gamma}$ and

$$
\kappa_{c}=\frac{N-2(\gamma+1)-\sqrt{(N-2(\gamma+1))^{2}-4 c}}{2} .
$$

If $\Omega \equiv B_{1}(0)$, then $\psi$ is a solution to the problem

$$
-\operatorname{div}\left(|x|^{-2 \gamma} \nabla \psi\right)=c \frac{\psi}{|x|^{2(\gamma+1)}}, \quad \psi \geq 0,\left.\quad \psi\right|_{\partial \Omega}=1 .
$$

Therefore we have the following theorem.

Theorem 5.5. Let $u_{0} \in L^{1}(\Omega)$ be a nonnegative function such that $u_{0} \neq 0$. Suppose that one of the following conditions holds:

(1) $\alpha=1, \lambda<\lambda_{N, \gamma}$ and $\int_{\Omega} u_{0}(x)|x|^{-\kappa_{c}} d x<\infty$,

(2) $1+\gamma>0,0<\alpha<\alpha_{1}=1-\frac{\gamma+1}{N-(\gamma+1)}$ and $\lambda>0$,

(3) $1+\gamma \leq 0,0<\alpha<1$ and $\lambda>0$.

Then the problem

$$
\left\{\begin{array}{l}
u_{t}-\operatorname{div}\left(|x|^{-2 \gamma} \nabla u\right)=\lambda \frac{u^{\alpha}}{|x|^{2(\gamma+1)}}, \quad u \geq 0 \quad \text { in } \Omega \times(0, T), \\
u=0 \text { on } \partial \Omega \times(0, T), \\
u(x, 0)=u_{1}(x) \text { in } \Omega,
\end{array}\right.
$$

has a weak positive minimal solution. 
Proof. The first case follows by using a similar argument to that in [B] $]$. For the reader's convenience we sketch the proof here. Let $u_{n}$ be the minimal solution to the truncated problem

$$
\left\{\begin{array}{l}
\frac{\partial u_{n}}{\partial t}-\operatorname{div}\left(|x|^{-2 \gamma} \nabla u_{n}\right)=\lambda \frac{u_{n}}{|x|^{2(\gamma+1)}+1 / n}, \quad u_{n} \geq 0 \quad \text { in } \Omega \times(0, T), \\
u_{n}=0 \quad \text { on } \partial \Omega \times(0, T), \quad u_{n}(x, 0)=T_{n}\left(u_{0}(x)\right) \quad \text { in } \Omega .
\end{array}\right.
$$

Let $p \in C^{2}(\mathbb{R})$ be a convex function satisfying $p(0)=p^{\prime}(0)=0$ which approximates $|\cdot|$. Taking $p^{\prime}\left(u_{n}\right) \psi$ as a test function in 56) we obtain

$$
\begin{aligned}
\int_{\Omega} u_{n}(x, t) \psi(x) d x+(c-\lambda) \int_{0}^{T} & \int_{\Omega} \frac{u_{n} \psi}{|x|^{2(\gamma+1)}+1 / n} d x d t \\
& \leq \int_{\Omega} T_{n}\left(u_{0}\right) \psi d x \leq \int_{\Omega} u_{0}(x) \psi(x) d x .
\end{aligned}
$$

Since $\left\{u_{n}\right\}$ is an increasing sequence, using the fact that $\psi>1$, we get the existence of $u(x, t)$ such that

$$
\int_{\Omega} u_{n}(x, t) \psi d x \nearrow \int_{\Omega} u(x, t) \psi d x<\infty
$$

and

$$
\int_{0}^{T} \int_{\Omega} \frac{u_{n}}{|x|^{2(\gamma+1)}+1 / n} d x d t \nearrow \int_{0}^{T} \int_{\Omega} \frac{u(x, t)}{|x|^{2(\gamma+1)}} d x d t<\infty .
$$

By setting $f(x, t)=u /|x|^{2(\gamma+1)} \in L^{1}(\Omega \times(0, T))$ and then using the approximation procedure we get the desired result.

To prove the other cases we set $u_{n}^{0}(x)=T_{n}\left(u_{0}(x)\right)$ and we assume that $\lambda=1$. Let $u_{n}$ be the minimal positive solution to the problem

$$
\left\{\begin{array}{l}
\frac{\partial u_{n}}{\partial t}-\operatorname{div}\left(|x|^{-2 \gamma} \nabla u_{n}\right)=\frac{u_{n}^{\alpha}}{|x|^{2(\gamma+1)}}, \quad u_{n} \geq 0 \quad \text { in } \Omega \times(0, T), \\
u_{n}=0 \text { on } \partial \Omega \times(0, T) \\
u_{n}(x, 0)=u_{n}^{0}(x) \quad \text { in } \Omega .
\end{array}\right.
$$

Assume that $\gamma+1>0$. Since $\alpha<\alpha_{1}$ we get the existence of $\beta>0$ such that

$$
0<\beta<2(\gamma+1), \quad 2(\gamma+1)-\frac{\alpha \beta}{2}<N(1-\alpha) .
$$

Let $\phi$ be the positive solution to the problem

$$
-\operatorname{div}\left(|x|^{-2 \gamma} \nabla \phi\right)=\frac{1}{|x|^{\beta}},\left.\quad \phi\right|_{\partial \Omega}=0
$$

notice that $\phi \in L^{\infty}(\Omega)$. Using $\phi$ as a test function in 58 we obtain

$$
\frac{d}{d t} \int_{\Omega} u_{n} \phi d x+\int_{\Omega} \frac{u_{n}}{|x|^{\beta}} d x=\int_{\Omega} \frac{u_{n}^{\alpha} \phi}{|x|^{2(\gamma+1)}} .
$$


Using the Hardy-Sobolev, Hölder and Young inequalities we get

$$
\frac{d}{d t} \int_{\Omega} u_{n} \phi d x+\int_{\Omega} \frac{u_{n}}{|x|^{\beta}} d x \leq \varepsilon \int_{\Omega} u_{n} \phi d x+\varepsilon \int_{\Omega} \frac{u_{n}}{|x|^{\beta}} d x+C(N, \gamma, \varepsilon),
$$

where $\varepsilon$ can be chosen small. Therefore we conclude that

$$
\frac{d}{d t} \int_{\Omega} u_{n} \phi d x \leq \varepsilon \int_{\Omega} u_{n} \phi d x+C
$$

Using the Gronwall lemma we find that

$$
\int_{\Omega} u_{n}(x, t) \phi(x) d x \leq c_{1} t+c_{2}, \quad \int_{\Omega} \frac{u_{n}}{|x|^{\beta}} d x<c_{2}<\infty
$$

where $c_{1}$ and $c_{2}$ depend only on $n, \gamma$ and $\int_{\Omega} u_{0}(x) d x$. Therefore we get the existence of a measurable function $u \geq 0$ such that $u_{n}(x, t) \uparrow u(x, t)$ for all $(x, t) \in \Omega \times(0, T)$,

$$
\int_{\Omega} u(x, t) \phi(x) d x<\infty . \quad \int_{0}^{T} \int_{\Omega} \frac{u(x, t)}{|x|^{\beta}} d x d t<\infty
$$

We set $f_{n}(x, t)=u_{n}^{\alpha}(x, t) /|x|^{2(\gamma+1)}$. By the Hölder and Young inequalities we obtain

$$
\begin{aligned}
\int_{0}^{T} \int_{\Omega} f_{n}(x, t) d x d t & \leq C(N, \gamma) T+\int_{0}^{T} \int_{\Omega} \frac{u_{n}(x, t)}{|x|^{\beta}} d x d t \\
& \leq C(N, \gamma) T+\int_{0}^{T} \int_{\Omega} \frac{u(x, t)}{|x|^{\beta}} d x d t<\infty
\end{aligned}
$$

Then using the dominated convergence theorem we conclude that $f_{n}(x, t) \uparrow f(x, t)=$ $u^{\alpha} /|x|^{2(\gamma+1)} \in L^{1}(\Omega \times(0, T))$. The result now follows by applying the same arguments as in $[\overline{\mathrm{BM}}]$ and $[\overline{\mathrm{Pr}}]$.

If $1+\gamma \leq 0$, then using $T_{k}\left(u_{n}\right)$ as a test function in 55 we get

$$
\begin{aligned}
& \frac{d}{d t} \int_{\Omega} \Theta_{k}\left(u_{n}\right) d x+\int_{\Omega}\left|\nabla T_{k}\left(u_{n}\right)\right|^{2}|x|^{-2 \gamma} d x=\int_{\Omega} \frac{T_{k}\left(u_{n}\right) u_{n}^{\alpha}}{|x|^{2(\gamma+1)}} d x \\
& \leq \int_{\Omega} T_{k}\left(u_{n}\right) u_{n}^{\alpha} d x \leq k^{1-\alpha}|\Omega|^{1-\alpha}\left(\int_{\Omega} T_{k}\left(u_{n}\right) u_{n} d x\right)^{\alpha}
\end{aligned}
$$

Since $\Theta_{k}(s) \geq \frac{1}{2} T_{k}(s) s$, we obtain

$$
\frac{d}{d t} \int_{\Omega} \Theta_{k}\left(u_{n}\right) d x+\int_{\Omega}\left|\nabla T_{k}\left(u_{n}\right)\right|^{2}|x|^{-2 \gamma} d x \leq 2^{\alpha} k^{1-\alpha}|\Omega|^{1-\alpha}\left(\int_{\Omega} \Theta_{k}\left(u_{n}\right) d x\right)^{\alpha} .
$$

We conclude by following the argument above. 


\section{Some remarks on existence of solutions in the case $1+\gamma \leq 0$}

Consider the problem

$$
\left\{\begin{array}{l}
u_{t}-\operatorname{div}\left(|x|^{-2 \gamma} \nabla u\right)=\lambda \frac{u}{|x|^{2(\gamma+1)}} \quad \text { in } \Omega \times(0, T), \\
u \geq 0 \quad \text { in } \Omega \times(0, T), \quad u=0 \quad \text { on } \partial \Omega \times(0, T), \\
u(x, 0)=u_{0}(x) \quad \text { in } \Omega, \quad u_{0} \in L^{2}(\Omega),
\end{array}\right.
$$

where we assume that $1+\gamma \leq 0$ and $\lambda>0$. In this case we have the following energy estimate:

$\int_{\Omega} u(x, t)^{2} d x+\int_{0}^{t} \int_{\Omega}|\nabla u|^{2}|x|^{-2 \gamma} d x d \tau=\lambda \int_{0}^{t} \int_{\Omega} \frac{u^{2}}{|x|^{2(\gamma+1)}} d x d \tau+\int_{\Omega} u_{0}(x)^{2} d x$.

Since $1+\gamma \leq 0$ we have

$$
\lambda \int_{0}^{t} \int_{\Omega} \frac{u^{2}}{|x|^{2(\gamma+1)}} d x d \tau \leq c(\Omega) \lambda \int_{0}^{t} \int_{\Omega} u^{2} d x d \tau .
$$

Using the Gronwall inequality and classical arguments to pass to the limit we are able to obtain the following result.

Theorem 6.1. If $1+\gamma \leq 0, \lambda>0$ and $u_{0} \in L^{2}(\Omega)$, then problem 60 has a unique solution $u \in L^{2}\left((0, T) ; \overline{\mathcal{D}}_{0, \gamma}^{1,2}(\Omega)\right) \cap C\left([0, T] ; L^{2}(\Omega)\right)$.

This is a particular case of the existence results in [DGP]. The details can be found there.

Remark 6.2. Notice that the result is independent of the relation between $\lambda$ and $\lambda_{N, \gamma}$ and this is the deep difference with respect to the behavior of problem (60) if $1+\gamma>0$, analyzed above. In this last case the existence is obtained only if $\lambda \leq \lambda_{N}, \gamma$. We have proved the nonexistence result in the previous section. The existence result for $\lambda \leq \lambda_{N, \gamma}$ is obtained by using the corresponding Hardy-Sobolev inequality which provides an energy estimate. We refer to [DGP] for details and extensions to the quasilinear framework.

\section{Appendix: Uniqueness result for the elliptic case}

We give a sketch of the proof of the uniqueness result for the elliptic case. We refer to [AP3] for more details.

The results which extend the classical regularity result by Weyl for the Laplace equation have been obtained by several authors, in particular, F. E. Browder [Bro] and L. Nirenberg $[\bar{N}]$ in the case of strongly elliptic equations with regular coefficients. On the other hand, there are results by J. Serrin $[\mathrm{S}]$ on nonuniqueness for discontinuous coefficients. In our problems the differential operator is either degenerate or with coefficients singular at 0 . Thus we can view the uniqueness result as an extension to these cases of the results quoted above about uniqueness (regularity). One of the main tools in the proof is the following result of Fourier analysis (we refer to [Gr, p. 127, Proposition 3.6.2] for a proof). We write $\mathcal{H}_{n}=\left\{\psi_{i} \mid\right.$ the spherical harmonics of degree $\left.n\right\}$. For the definition and properties of the spherical harmonics we refer to the book by E. M. Stein [St], pp. 68-77. 
Lemma 7.1. Assume that $\phi$ is a $2 k$-times continuously differentiable function in $\bar{B}$ with $k \geq[N / 4]+1$, where $[\cdot]$ is the integer part function. Let

$$
c_{i}(r)=\int_{S^{N-1}} \phi(r, \sigma) \psi_{i}(\sigma) d \sigma, \quad Q_{n}(r, \sigma)=\sum_{\chi\left(\psi_{i}\right)=n} c_{i}(r) \psi_{i}(\sigma),
$$

where $\chi\left(\psi_{i}\right)=n$ means that the degree of $\psi_{i}$ is $n$. Then

$$
\left|Q_{n}(r, \sigma)\right|^{2} \leq \mu(N)\left\|\Delta_{0}^{k} \phi\right\|^{2} n^{N-4 k-2} \leq C(N, k, \phi) n^{N-4 k-2},
$$

where $\Delta_{0}^{k}$ denotes the $k$-times iterated Laplace-Beltrami operator, $\left\|\Delta_{0}^{k} \phi\right\|^{2}=$ $\int_{S^{N-1}}\left|\left(\Delta_{0}^{k} \phi\right)(r, \sigma)\right|^{2} d \sigma \mid$ and $\mu(N)$ is a positive constant depending on $N$. Moreover

$$
\phi(r, \sigma)=\sum_{n=0}^{\infty} Q_{n}(r, \sigma)=\sum_{n=0}^{\infty} \sum_{\left(\psi_{i}\right)=n} c_{i}(r) \psi_{i}(\sigma)=\sum_{i=0}^{\infty} c_{i}(r) \psi_{i}(\sigma)
$$

and the convergence is uniform in $\bar{B}$.

Let $\Omega$ be a bounded domain such that $0 \in \Omega$. We begin by the following definition.

Definition 7.2. Let $f \in L^{1}(\Omega)$ and $u \in L^{1}\left(|x|^{-2 \gamma-1} d x, \Omega\right)$. We say that $u$ is a weak solution to the problem

$$
-L_{\gamma}(u) \equiv-\operatorname{div}\left(|x|^{-2 \gamma} \nabla u\right)=f
$$

if for all $\phi \in C_{0}^{\infty}(\Omega)$ we have

$$
\int_{\Omega} u\left(-L_{\gamma}(\phi)\right) d x=\int_{\Omega} f \phi d x
$$

The main result in this appendix is the following theorem.

Theorem 7.3. Let $-\infty<\gamma<(N-2) / 2$. Assume that $u \in L^{1}\left(|x|^{-2 \gamma-1} d x, \Omega\right)$ is a weak solution to

$$
-\operatorname{div}\left(|x|^{-2 \gamma} \nabla u\right)=0
$$

in the sense of Definition 7.2 Then $u \in C^{\varepsilon}(\Omega) \cap \mathcal{D}_{\gamma, \text { loc }}^{1,2}$, where $\varepsilon$ depends on $N$ and $\gamma$ and

$\mathcal{D}_{\gamma, \text { loc }}^{1,2}=\left\{u: \int_{K}|\nabla u|^{2}|x|^{-2 \gamma} d x+\int_{K}|u|^{2}|x|^{-2 \gamma} d x<\infty, \forall K \subset \subset \Omega, K\right.$ compact $\}$.

Proof. Since $|x|^{-2 \gamma} \in C^{\infty}(\Omega \backslash\{0\})$, it follows that $u \in C^{\infty}(\Omega \backslash\{0\})$ by the Weyl lemma for regular coefficients. See $[\overline{\mathrm{Bro}}]$ and $[\overline{\mathrm{N}}]$. Hence we just have to prove the regularity of $u$ at 0 . Without loss of generality we can assume that $B_{1}(0) \subset \Omega$. The idea of the proof is to consider a convenient Fourier expansion of the solution and to show that the coefficients corresponding to the singular radial part are zero. We divide the proof into several steps.

Step 1. Fourier expansion. Since $u$ is regular for $r>0$, expanding it in terms of $\left\{\psi_{i}\right\}$, the eigenfunctions of the Laplace-Beltrami operator $-\Delta_{\sigma}$ in $S^{N-1}$, we obtain

$$
u(r, \sigma)=\sum_{i \geq 0} f_{i}(r) \psi_{i}(\sigma), \quad \text { where } \quad f_{i}(r)=\left.\int_{S^{N-1}} u\right|_{\partial B_{r}}(r, \sigma) \psi_{i}(\sigma) d \sigma .
$$


Since $u$ is a classical solution of 63 for $r \in[\varepsilon, 1]$, we get

$$
\sum_{i \geq 0}\left\{f_{i}^{\prime \prime}(r)+\frac{N-1-2 \gamma}{r} f_{i}^{\prime}(r)-\frac{c_{i}}{r^{2}} f_{i}\right\} \psi_{i}(\sigma)=0 .
$$

Hence

$$
f_{i}^{\prime \prime}(r)+\frac{N-1-2 \gamma}{r} f_{1}^{\prime}(r)-\frac{c_{i}}{r^{2}} f_{i}=0 \quad \text { for all } i .
$$

Therefore

$$
f_{0}(r)=A_{0} r^{2(\gamma+1)-N}+B_{0}, \quad f_{i}(r)=A_{i} r^{\alpha_{i}}+B_{i} r^{\beta_{i}}
$$

where

$$
\begin{aligned}
\alpha_{i} & =\frac{-\left[N-2(\gamma+1)+\sqrt{(N-2(\gamma+1))^{2}+4 c_{i}}\right]}{2}, \\
\beta_{i} & =\frac{\sqrt{N-2(\gamma+1))^{2}+4 c_{i}}-(N-2(\gamma+1))}{2}, \\
c_{i} & =i(N+i-2) \quad \text { for } i \geq 0,
\end{aligned}
$$

and

$$
\left\{\begin{array}{l}
A_{0}(\varepsilon)=\frac{\varepsilon^{N-2(\gamma+1)}\left(f_{0}(\varepsilon)-f_{0}(1)\right)}{1-\varepsilon^{N-2(\gamma+1)}} \\
B_{0}(\varepsilon)=\frac{f_{0}(1)-\varepsilon^{N-2(\gamma+1)} f_{0}(\varepsilon)}{1-\varepsilon^{N-2(\gamma+1)}}
\end{array}\right.
$$

and

$$
\left\{\begin{array}{l}
A_{i}(\varepsilon)=\frac{\varepsilon^{-\alpha_{i}} f_{i}(\varepsilon)-\varepsilon^{\beta_{i}-\alpha_{i}} f_{i}(1)}{1-\varepsilon^{\beta_{i}-\alpha_{i}}}, \\
B_{i}(\varepsilon)=\frac{f_{i}(1)-\varepsilon^{-\alpha_{i}} f_{i}(\varepsilon)}{1-\varepsilon^{\beta_{i}-\alpha_{i}}},
\end{array}\right.
$$

for $i \geq 1$, where for all $\varepsilon>0$ we write

$$
f_{i}(\varepsilon)=\left.\int_{S^{N-1}} u\right|_{\partial B_{\varepsilon}}(\varepsilon, \sigma) \psi_{i}(\sigma) d \sigma .
$$

Notice that $\beta_{i}>0$ if $i>0$. We conclude that for $r \in(\varepsilon, 1)$ we have

$$
\begin{aligned}
u(r, \sigma)= & \frac{f_{0}(1)-\varepsilon^{N-2(\gamma+1)} f_{0}(\varepsilon)}{1-\varepsilon^{N-2(\gamma+1)}+\frac{\varepsilon^{N-2(\gamma+1)}\left(f_{0}(\varepsilon)-f_{0}(1)\right)}{1-\varepsilon^{N-2(\gamma+1)}} r^{2(\gamma+1)-N}} \\
& +\sum_{i \geq 1}\left\{\frac{\varepsilon^{-\alpha_{i}} f_{i}(\varepsilon)-\varepsilon^{\beta_{i}-\alpha_{i}} f_{i}(1)}{1-\varepsilon^{\beta_{i}-\alpha_{i}}} r^{\alpha_{i}}+\frac{f_{i}(1)-\varepsilon^{-\alpha_{i}} f_{i}(\varepsilon)}{1-\varepsilon^{\beta_{i}-\alpha_{i}}} r^{\beta_{i}}\right\} \psi_{i}(\sigma) .
\end{aligned}
$$

Step 2. Decay of the coefficients. We claim that $\left|\varepsilon^{N-2(\gamma+1)} f_{i}(\varepsilon)\right| \rightarrow 0$. Notice that, as a consequence, $\left|\varepsilon^{-\alpha_{i}} f_{i}(\varepsilon)\right| \rightarrow 0$ as $\varepsilon \rightarrow 0$ for all $i \geq 0$. We first assume that $i \geq 1$. Let 
$\phi \in C_{0}^{\infty}(B)$. Since $u$ is a solution to $[63$ and a classical solution for $|x|>0$, we obtain

$$
\begin{aligned}
0=\int u\left(L_{\gamma}(\phi)\right) d x=\lim _{\varepsilon \rightarrow 0} \int_{|x|>\varepsilon} u L_{\gamma}(\phi) d x \\
=\lim _{\varepsilon \rightarrow 0}\left\{\int_{|x|>\varepsilon} \phi L_{\gamma}(u)-\int_{|x|=\varepsilon}|x|^{-2 \gamma} u\langle\nabla \phi, x /|x|\rangle d \sigma\right. \\
\left.\quad+\int_{|x|=\varepsilon}|x|^{-2 \gamma} \phi\langle\nabla u, x /|x|\rangle d \sigma\right\} .
\end{aligned}
$$

Since $L_{\gamma} u(x)=0$ for $|x| \geq \varepsilon$, by setting $\phi(x)=g(r) \psi_{i_{0}}(\sigma)$, where $g \in C^{\infty}([0, a))$, $a<1, g(0)=1$, with supp $g \subset[0, a)$, and using the fact that for $x=r \sigma$ we have $x \nabla v=r \partial v / \partial r$, and by the orthogonality of the spherical harmonics, we obtain

$$
\begin{aligned}
0=\lim _{\varepsilon \rightarrow 0}\left\{-\varepsilon^{N-1-2 \gamma} \int_{S^{N-1}}\left(A_{i_{0}}(\varepsilon) \varepsilon^{\alpha_{i_{0}}}+B_{i_{0}}(\varepsilon) \varepsilon^{\beta_{i_{0}}}\right) g^{\prime}(\varepsilon) \psi_{i_{0}}^{2} d \sigma\right. \\
\left.+\varepsilon^{N-2(\gamma+1)} \int_{S^{N-1}}\left(\alpha_{i_{0}} A_{i_{0}}(\varepsilon) \varepsilon^{\alpha_{i_{0}}}+\beta_{i_{0}} B_{i_{0}}(\varepsilon) \varepsilon^{\beta_{i_{0}}}\right) g(\varepsilon) \psi_{i_{0}}^{2} d \sigma\right\} .
\end{aligned}
$$

Hence we conclude that

$$
\begin{aligned}
\lim _{\varepsilon \rightarrow 0}\left\{\varepsilon ^ { N - 1 - 2 \gamma } \left(A_{i_{0}}(\varepsilon) \varepsilon^{\alpha_{i_{0}}}\right.\right. & \left.+B_{i_{0}}(\varepsilon) \varepsilon^{\beta_{i_{0}}}\right) g^{\prime}(\varepsilon) \\
& \left.-\varepsilon^{N-2(\gamma+1)}\left(\alpha_{i_{0}} A_{i_{0}}(\varepsilon) \varepsilon^{\alpha_{i_{0}}}+\beta_{i_{0}} B_{i_{0}}(\varepsilon) \varepsilon^{\beta_{i_{0}}}\right) g(\varepsilon)\right\}=0 .
\end{aligned}
$$

Therefore

$$
\begin{aligned}
0= & \lim _{\varepsilon}\left\{\varepsilon^{N-1-2 \gamma}\left(\left[f_{i_{0}}(\varepsilon)-\varepsilon^{\beta_{i_{0}}} f_{i_{0}}(1)\right]+\left[f_{i_{0}}(1)-\varepsilon^{-\alpha_{i_{0}}} f_{i_{0}}(\varepsilon)\right] \varepsilon^{\beta_{i_{0}}}\right) g^{\prime}(\varepsilon)\right. \\
& \left.-\varepsilon^{N-2(\gamma+1)}\left(\alpha_{i_{0}}\left[f_{i_{0}}(\varepsilon)-\varepsilon^{\beta_{i_{0}}} f_{i_{0}}(1)\right]+\beta_{i_{0}}\left[f_{i_{0}}(1)-\varepsilon^{-\alpha_{i_{0}}} f_{i_{0}}(\varepsilon)\right] \varepsilon^{\beta_{i_{0}}}\right) g(\varepsilon)\right\} .
\end{aligned}
$$

Hence

$$
\begin{aligned}
\lim _{\varepsilon \rightarrow 0}\left\{\varepsilon^{N-1-2 \gamma}\left(f_{i_{0}}(\varepsilon)-\varepsilon^{\beta_{i_{0}}-\alpha_{i_{0}}} f_{i_{0}}(\varepsilon)\right) g^{\prime}(\varepsilon)\right. & \\
& \left.-\varepsilon^{N-2(\gamma+1)}\left(\alpha_{i_{0}} f_{i_{0}}(\varepsilon)-\beta_{i_{0}} \varepsilon^{\beta_{i_{0}}-\alpha_{i_{0}}} f_{i_{0}}(\varepsilon)\right) g(\varepsilon)\right\}=0 .
\end{aligned}
$$

Finally, we obtain

$$
\lim _{\varepsilon \rightarrow 0}\left\{\varepsilon^{N-2(\gamma+1)} f_{i_{0}}(\varepsilon)\left[\left(\varepsilon-\varepsilon^{\beta_{i_{0}}-\alpha_{i_{0}}+1}\right) g^{\prime}(\varepsilon)-\left(\alpha_{i_{0}}-\beta_{i_{0}} \varepsilon^{\beta_{i_{0}}-\alpha_{i_{0}}}\right) g(\varepsilon)\right]\right\}=0 .
$$

Since

$$
\left(\varepsilon-\varepsilon^{\beta_{i_{0}}-\alpha_{i_{0}}+1}\right) g^{\prime}(\varepsilon)-\left(\alpha_{i_{0}}-\beta_{i_{0}} \varepsilon^{\beta_{i_{0}}-\alpha_{i_{0}}}\right) g(\varepsilon) \rightarrow-\alpha_{i_{0}} \quad \text { as } \varepsilon \rightarrow 0,
$$

we obtain the claim in the case $i \geq 1$.

If $i=0$, then by setting $\phi(x)=g(r)$ and using the same argument as above we obtain

$$
\lim _{\varepsilon \rightarrow 0}\left\{\varepsilon^{N-2(\gamma+1)} f_{0}(\varepsilon)\left[\left(\varepsilon-\varepsilon^{N-2(\gamma+1)+1}\right) g^{\prime}(\varepsilon)+(N-2(\gamma+1)) g(\varepsilon)\right]\right\}=0 .
$$

Then we conclude that $\lim _{\varepsilon \rightarrow 0}\left|\varepsilon^{N-2(\gamma+1)} f_{0}(\varepsilon)\right|=0$ and the claim is proved. 
Step 3. Density argument and conclusion of the proof. We set

$$
v(r, \sigma)=\sum_{i \geq 0} f_{i}(1) r^{\beta_{i}} \psi_{i}(\sigma),
$$

which is the unique solution in the space $C^{\varepsilon}(\Omega) \cap \mathcal{D}_{\gamma, \text { loc }}^{1,2}$ to the Dirichlet problem with boundary data $v=u$ on the unit sphere. We will prove that $u=v$ a.e. in $B$. Let $\phi \in$ $C_{0}^{\infty}(\Omega)$ be such that $\phi(r, \sigma)=g_{i_{0}}(r) \psi_{i_{0}}(\sigma)$, where $g_{i_{0}} \in C_{0}^{\infty}([0,1))$ and $i_{0} \geq 1$. Then

$$
\int_{B} v(x) \phi(x)|x|^{-2 \gamma-1} d x=f_{i_{0}}(1) \int_{0}^{1} g_{i_{0}}(r) r^{N-2(\gamma+1)+\beta_{i_{0}}} d r .
$$

On the other hand, by orthogonality we have

$$
\begin{aligned}
I_{i_{0}} \equiv & \int_{B} u(x) \phi(x)|x|^{-2 \gamma-1} d x=\lim _{\varepsilon \rightarrow 0} \int_{|x|>2 \varepsilon} u(x) \phi(x)|x|^{-2 \gamma-1} d x \\
= & \lim _{\varepsilon \rightarrow 0} \int_{2 \varepsilon}^{1} \int_{S^{N-1}} \sum_{i=0}^{\infty}\left(A_{i}(\varepsilon) r^{\alpha_{i}}+B_{i}(\varepsilon) r^{b_{i}}\right) \psi_{i}(\sigma) \phi(r, \sigma) d \sigma \\
= & \lim _{\varepsilon \rightarrow 0} \int_{2 \varepsilon}^{1}\left(A_{i}(\varepsilon) r^{\alpha_{i}}+B_{i}(\varepsilon) r^{b_{i}}\right) g_{i_{0}}(r) r^{N-2(\gamma+1)} d r \\
= & \lim _{\varepsilon \rightarrow 0} \int_{2 \varepsilon}^{1}\left\{\frac{\varepsilon^{-\alpha_{i_{0}}} f_{i_{0}}(\varepsilon)-\varepsilon^{\beta_{i_{0}}-\alpha_{i_{0}}} f_{i_{0}}(1)}{1-\varepsilon^{\beta_{i_{0}}-\alpha_{i_{0}}}} r^{\alpha_{i_{0}}}\right\} g_{i_{0}}(r) r^{N-2(\gamma+1)} d r \\
& +\lim _{\varepsilon \rightarrow 0} \int_{2 \varepsilon}^{1}\left\{\frac{f_{i_{0}}(1)-\varepsilon^{-\alpha_{i_{0}}} f_{i_{0}}(\varepsilon)}{1-\varepsilon^{\beta_{i_{0}}-\alpha_{i_{0}}}} r^{\beta_{i_{0}}}\right\} g_{i_{0}}(r) r^{N-2(\gamma+1)} d r .
\end{aligned}
$$

Since by step $2,\left|\varepsilon^{N-2(\gamma+1)} f_{i_{0}}(\varepsilon)\right| \rightarrow 0$ as $\varepsilon \rightarrow 0$, we conclude that

$$
I_{i_{0}}=f_{i_{0}}(1) \int_{0}^{1} g_{i_{0}}(r) r^{N-2(\gamma+1)+\beta_{i_{0}}} d r .
$$

If $i_{0}=0$, then choosing $\phi(x)=g_{0}(r) \in C_{0}^{\infty}([0,1))$, by the same argument as above, we obtain

$$
I_{0}=f_{0}(1) \int_{0}^{1} g_{0}(r) r^{N-2(\gamma+1)} d r .
$$

Therefore we conclude that for all $\phi \in C_{0}^{\infty}\left(B_{1}(0)\right)$ of the form $\phi(x)=g_{i}(r) \psi_{i}(\sigma)$ we have

$$
\int_{B} u(x) \phi(x)|x|^{-2 \gamma-1} d x=\int_{B} v \phi(x)|x|^{-2 \gamma-1} d x .
$$

Notice that 66 holds for all $\phi \in C(\bar{B})$ such that $\phi(r, \sigma)=g(r) \psi_{i}(\sigma)$, where $g \in$ $C([0,1])$. We set

$$
\mathcal{F}=\left\{\sum_{k=0}^{n} g_{k}(r) \psi_{k}(\sigma) \mid n \in \mathbb{N} \text { and } g_{k} \in C^{\infty}([0,1]) \text { for } 0 \leq k \leq n\right\} .
$$

Then for all $\phi \in \mathcal{F}$ we have

$$
\int_{B} u(x) \phi(x)|x|^{-2 \gamma-1} d x=\int_{B} v \phi(x)|x|^{-2 \gamma-1} d x .
$$


Therefore using Lemma 7.1 we get the density of $\mathcal{F}$ in $C_{0}^{\infty}\left(B_{1}(0)\right)$ with respect to the uniform convergence, hence

$$
\int_{B} u(x) \phi(x)|x|^{-2 \gamma-1} d x=\int_{B} v \phi(x)|x|^{-2 \gamma-1} d x
$$

for all $\phi \in C_{0}^{\infty}\left(B_{1}(0)\right)$. Since $u(x)|x|^{-2 \gamma-1}, v(x)|x|^{-2 \gamma-1} \in L^{1}\left(B_{1}(0)\right)$ we obtain

$$
u(x)=v(x) \quad \text { for a.e. } x \in B_{1}(0) .
$$

As a consequence (setting $d \mu=|x|^{-2 \gamma-1} d x$ ) we get the next result.

Corollary 7.4. Let $f \in L^{1}(\Omega)$. Then the problem

$$
-L_{\gamma}(u)=f,\left.\quad u\right|_{\partial \Omega}=0,
$$

has a unique weak solution $u \in L^{1}(d \mu, \Omega)$ such that

$$
\|u\|_{L^{1}(d \mu, \Omega)} \leq C\|f\|_{L^{1}(\Omega)},
$$

where $C$ depends on $N, \gamma$ and $\Omega$. Moreover, if $\gamma+1>0$ we have

$$
\|u\|_{L^{1}(\Omega)} \leq C\|f\|_{L^{1}(\Omega)} .
$$

Acknowledgments. The third author wants to thank Professor Haïm Brezis for his encouragement to prove the uniqueness results in this work, and Professor Cristian E. Gutiérrez for his helpful comments about the parabolic Harnack inequality for degenerate and singular linear parabolic equations.

\section{References}

[AP1] Abdellaoui, B., Peral, I.: Some results for semilinear elliptic equations with critical potential. Proc. Roy. Soc. Edinburgh Sect. A 132, 1-24 (2002) Zbl 1014.35023 MR 2002k:35106

[AP2] Abdellaoui, B., Peral, I.: Existence and nonexistence results for quasilinear elliptic equations involving the $p$-Laplacian with a critical potential. Ann. Mat. Pura Appl. 182, 247$270(2003)$

[AP3] Abdellaoui, B., Peral, I.: Some results for quasilinear elliptic equations related to some Caffarelli-Kohn-Nirenberg inequalities. Comm. Pure Appl. Anal. 2, 539-566 (2003)

[AP4] Abdellaoui, B., Peral, I.: Hölder continuity and Harnack inequality for quasilinear parabolic equations with weight. Preprint

[AgP] Aguilar, J. A., Peral, I.: Global behaviour of the Cauchy problem for some critical nonlinear parabolic equations. SIAM J. Math. Anal. 31, 1270-1294 (2000)

[BG] Baras, P., Goldstein, J.: The heat equation with a singular potential. Trans. Amer. Math. Soc. 284, 121-139 (1984) Zbl 0556.35063 MR 85f:35099

[BBC] Benilan, P., Brezis, H., Crandall, M.: A semilinear equation in $L^{1}\left(R^{N}\right)$. Ann. Scuola Norm. Sup. Pisa Cl. Sci. (4) 2, 523-555 (1975) Zbl 0314.35077 MR 52 \#11299

[BM] Blanchard, D., Murat, F.: Renormalised solutions of nonlinear parabolic problems with $L^{1}$ data: existence and uniqueness. Proc. Roy. Soc. Edinburgh Sect. A 127, 1137-1152 (1997) Zbl 0895.35050 MR 98i:35096

[BCr] Brezis, H., Crandall, M.: Uniqueness of solutions of the initial-value problem for $u_{t}-\Delta \varphi(u)=0$. J. Math. Pures Appl. (9) 58, 153-163 (1979) Zbl 0408.35054 MR 80e:35029 
[BC] Brezis, H., Cabré, X.: Some simple nonlinear PDE's without solutions. Boll. Unione Mat. Ital. Sez. B Artic. Ric. Mat. (8) 1, 223-262 (1998) Zbl 0907.35048 MR 99j:35001

[Bro] Browder, F. E.: On the regularity properties of solutions of elliptic differential equations. Comm. Pure Appl. Math. 9, 351-361 (1956) Zbl 0070.09601 MR 19,862a

[CM] Cabré, X., Martel, Y.: Existence versus instantaneous blow-up for linear heat equations with singular potentials. C. R. Acad. Sci. Paris Sér. I Math. 329, 973-978 (1999) Zbl 0940.35105 MR 2000j:35117

[CKN] Caffarelli, L., Kohn, R., Nirenberg, L.: First order interpolation inequalities with weights. Compositio Math. 53, 259-275 (1984) Zbl 0563.46024 MR 86c:46028

[CW] Catrina, F., Wang, Z.-Q.: On the Caffarelli-Kohn-Nirenberg inequalities: sharp constants, existence (and nonexistence), and symmetry of extremal functions. Comm. Pure Appl. Math. 54, 229-258 (2001) Zbl pre01592013 MR 2001k:35028

[ChF] Chiarenza, F. M., Frasca, M.: Boundedness for the solutions of a degenerate parabolic equation, Applicable Anal. 17, 243-261 (1984) Zbl 0522.35059 MR 86c:35081

[ChS] Chiarenza, F. M., Serapioni, R. P.: A Harnack inequality for degenerate parabolic equations. Comm. Partial Differential Equations 9, 719-749(1984) Zbl 0546.35035 MR 86c:35082

[DGP] Dall'Aglio, A., Giachetti, D., Peral, I.: Results on parabolic equations related to CaffarelliKohn-Nirenberg inequalities. Preprint

[F] Fujita, H.: On some nonexistence and nonuniqueness theorems for nonlinear parabolic equations. In: Nonlinear Functional Analysis (Chicago, IL, 1968), Proc. Sympos. Pure Math. 18, Part 1, Amer. Math. Soc., Providence, RI, 105-113 (1970) Zbl 0228.35048 MR 42 \#4888

[GP] García Azorero, J., Peral, I.: Hardy inequalities and some critical elliptic and parabolic problems. J. Differential Equations 144, 441-476 (1998) Zbl 0918.35052

[Gr] Groemer, H.: Geometric Applications of Fourier Series and Spherical Harmonics. Encyclopedia Math. Appl. 61, Cambridge Univ. Press (1996) Zbl 0877.52002 MR 97j:52001

[GW] Gutiérrez, C. E., Wheeden, R. L.: Mean value and Harnack inequalities for degenerate parabolic equations. Colloq. Math. 60/61, 157-194 (1990) Zbl 0785.35057 MR 92d:35025

[N] Nirenberg, L.: Remarks on strongly elliptic partial differential equations. Comm. Pure Appl. Math. 8, 648-674 (1955) Zbl 0067.07602 MR 17,742d

[PV] Peral, I., Vázquez, J. L.: On the stability or instability of the singular solution of the semilinear heat equation with exponential reaction term. Arch. Rat. Mech. Anal. 129, 201-224 (1995) Zbl 0821.35080 MR 96b:35023

[P] Picone, M.: Sui valori eccezionali di un parametro da cui dipende una equazione differenziale lineare ordinaria del secondo ordine. Ann. Scuola Norm. Sup. Pisa 11, 1-144 (1910) JFM 41.0351.01

[Pi] Pierre, M.: Uniqueness of the solutions of $u_{t}-\Delta \varphi(u)=0$ with initial datum a measure. Nonlinear Anal. 6, 175-187 (1982) Zbl 0484.35044 MR 83h:35062

[Pr] Prignet, A.: Existence and uniqueness of "entropy" solutions of parabolic problems with $L^{1}$ data. Nonlinear Anal. 28, 1943-1954 (1997) Zbl 0909.35075 MR 98b:35085

[S] Serrin, J.: Pathological solutions of elliptic differential equations. Ann. Scuola Norm. Sup. Pisa Cl. Sci. (3) 18, 385-387 (1964) Zbl 0142.37601 MR 30 \#335

[St] Stein, E. M.: Singular Integrals and Differentiability Properties of Functions. Princeton Univ. Press, Princeton, NJ (1970) Zbl 0207.13501 MR 44 \#7280 